IZA DP No. 6298

Insurance versus Savings for the Poor:

Why One Should Offer Either Both or None

Andreas Landmann

Björn Vollan

Markus Frölich

January 2012 


\title{
Insurance versus Savings for the Poor: Why One Should Offer Either Both or None
}

\author{
Andreas Landmann \\ University of Mannheim \\ Björn Vollan \\ University of Mannheim \\ and $Z M T$ \\ Markus Frölich \\ University of Mannheim, \\ ZEW and IZA
Discussion Paper No. 6298
January 2012 \\ IZA
P.O. Box 7240
53072 Bonn
Germany \\ Phone: +49-228-3894-0
Fax: +49-228-3894-180
E-mail: iza@iza.org
}

\begin{abstract}
Any opinions expressed here are those of the author(s) and not those of IZA. Research published in this series may include views on policy, but the institute itself takes no institutional policy positions.

The Institute for the Study of Labor (IZA) in Bonn is a local and virtual international research center and a place of communication between science, politics and business. IZA is an independent nonprofit organization supported by Deutsche Post Foundation. The center is associated with the University of Bonn and offers a stimulating research environment through its international network, workshops and conferences, data service, project support, research visits and doctoral program. IZA engages in (i) original and internationally competitive research in all fields of labor economics, (ii) development of policy concepts, and (iii) dissemination of research results and concepts to the interested public.
\end{abstract}

IZA Discussion Papers often represent preliminary work and are circulated to encourage discussion. Citation of such a paper should account for its provisional character. A revised version may be available directly from the author. 


\section{ABSTRACT}

\section{Insurance versus Savings for the Poor: Why One Should Offer Either Both or None*}

This paper analyzes data from a novel field experiment designed to test the impact of two different insurance products and a secret saving device on solidarity in risk-sharing groups among rural villagers in the Philippines. Risk is simulated by a lottery. Risk-sharing is possible in solidarity groups of three and insurance is introduced via less risky lotteries. Our main hypothesis is that formal market-based products lead to lower voluntary transfers among network members. We also test for the persistence of this crowding-out of solidarity. We find evidence for a reduction of solidarity by insurance if shocks are observable. Depending on insurance design, there is also evidence for persistence of this effect even if insurance is removed. Simulations using our regression results show that the benefits of insurance are completely offset by the reduction in transfers. However, if secret saving is possible solidarity is very low in general and there is no crowding out effect of insurance. This suggests that introducing formal insurance is not as effective as it is hoped for when the monetary situation can be closely monitored, but that it might be a very important complement when savings inhibit observing financial resources. The implication for policy is that microsavings should be offered simultaneously with microinsurance.

JEL Classification: C93, O12, Z13

Keywords: insurance, savings, informal risk sharing, crowding out, field lab experiment, Philippines

Corresponding author:

Andreas Landmann

Department of Economics

University of Mannheim

L7, 3-5

68131 Mannheim

Germany

E-mail: andreas.landmann@uni-mannheim.de

\footnotetext{
* We gratefully acknowledge financial and organizational support by 'Deutsche Gesellschaft für Internationale Zusammenarbeit' (GIZ) and its 'Microinsurance Innovations Program for Social Security' in the Philippines (GIZ-MIPSS). This research was also supported by the Research Center (SFB) 884 "Political Economy of Reforms" (Project B5), funded by the German Research Foundation (DFG). We thank Boris Branisa, Stefan Dercon, Dirk Engelmann, Xavier Giné, Simone Gobien, Marcela Ibanez, Dean Karlan, Niels Kemper, Paolo Masella, Katharina Michaelowa, Robert Poppe, Sebastian Prediger, Philip Zahn, and conference participants in Barcelona (International Meeting of Behavioural and Experimental Economics 2011), Madison (8th Midwest International Economic Development Conference), Groningen (2nd European Research Conference on Microfinance), Berlin (AEL Conference 2011), Paris (DIAL Development Conference 2011), Amsterdam (10th EUDN PhD Workshop), Oslo (26th EEA Congress) and Rio de Janeiro (7th International Microinsurance Conference) for helpful and valuable comments. Many thanks also to Philip Saliba and the team of local assistants for excellent support in the field.
} 


\section{Introduction}

A large majority of the population in the world's poorest countries is without formal insurance. ${ }^{1}$ Shocks such as natural catastrophes, illnesses, accidents, economic crises, unemployment or crime, droughts and floods, just to mention a few, destroy the economic basis of countless households. As a response, informal transfers within networks of friends, neighbors and relatives are important in the management of these income fluctuations, with transfers consisting of e.g. loans, monetary gifts, goods (such as food) or labor. These support schemes allow households to spread the effects of income shocks throughout their network or village. In this sense, the mutual support in case of a shock is an informal insurance mechanism relying on intrinsic motivation to act solidarily. ${ }^{2}$ This intrinsic motivation is based on friendship and kinship, altruism, inequity aversion or reciprocity (Barr and Genicot 2008).

There is ample evidence for the importance of such mechanisms in developing countries (e.g. see (Morduch 1999; Fafchamps 2008). Also on the Philippines risk-sharing networks play a major role and respondents may raise funds through gifts and loans (Fafchamps and Lund 2003), where loans are often zero-interest or do not have to be repaid fully (Fafchamps and Gubert 2007a). However, if other members are also suffering income shocks (covariate risk), it is more difficult for respondents to raise funds via informal ways. Furthermore, mutual insurance does not appear to take place at the village level; instead, households receive help primarily through networks of friends, relatives and those living close (Fafchamps and Lund 2003; Fafchamps and Gubert 2007b). Other evidence from developing countries around the globe suggests that informal insurance only smoothes a fraction of income shocks (Townsend 1994; Morduch 1999). ${ }^{3}$ Moreover, some do not regard these transfers as genuine risk-sharing. Platteau (1997) for example argues that donors in fact often expect a return for their payment instead of truly internalizing the spirit of mutual insurance. Ultimately, effectiveness of risk sharing rests on the willingness of those who were lucky to look after the less favored. Many unlucky remain excluded.

\footnotetext{
${ }^{1}$ Besides social security, only between $0.3 \%$ (Africa), $2.7 \%$ (Asia) and $7.8 \%$ (The Americas) of the target population in the 100 poorest countries is covered with formal insurance available to the poor (Roth, McCord, and Liber 2007, pp.15-19). Similarly, Banerjee and Duflo (2007) report that less than 6\% of the extremely poor are covered by any kind of health insurance. Also, coverage with formal savings is rather low in developing countries. According to Banerjee and Duflo (2007) the fraction of people with savings account is below $14 \%$.

2 The ILO Micro Insurance Compendium (Churchill 2006, p. 34) also mentions informal group-based mechanisms (burial societies etc.) as informal insurance. However, this already is a step towards formal mechanisms with an explicit obligation to pay contributions in order to receive benefits. We mean more flexible and non-contractual arrangements.

${ }^{3}$ Morduch (1999) summarizes some literature and concludes: "Most informal insurance mechanisms are typically weak and often provide only inadequate protection to poor households" (Morduch 1999, p. 188).
} 
These imperfections and drawbacks of informal mechanisms have made people consider how to remedy the situation for a long time. Some countries introduced universal insurance for some risks, e.g. free health insurance in India. Yet, even though public facilities should (at least by law) be for free under these schemes there have often been problems with low compliance, understaffing, corruption, quality of care as well as a high fiscal burden, which often led people to attend private clinics. Consequently, substantial interest in private insurance products remains (e.g. weather insurance, health, life). Especially the recent rise of microcredit and -saving concepts has led governments, financial institutions, NGOs and mutual benefit associations to the question: can we apply these new concepts to insurance, designing products especially suited for poor clients? In this spirit, many microinsurance initiatives are currently being launched and several pilot schemes are already in the field. Despite this effort, demand for microinsurance is so far still very low (Xavier Giné and Yang 2009; Cole et al. 2009; Ito and Kono 2010) and practitioners are working to implement an affordable insurance design that complements traditional informal risk sharing schemes. However, the possible effects of formal microinsurance on informal mechanisms are still an open question.

There are good reasons to believe that formal insurance will crowd out informal insurance and reduce solidarity, and that a flawed formal microinsurance system could even have negative overall effects on economic stability under certain circumstances (e.g. dependent on the fraction of insured people, strength of network, etc.). It is well established in the economic and psychological literature (Bowles 2008) that market-based mechanisms can crowd-out intrinsic pro-social behavior, and the introduction of formal insurance schemes can similarly reduce pro-social behavior. Instead of relying on the intrinsically motivated solidarity payments individuals may simply pay for formal insurance in order obtain more security. At least two of the causes for crowding-out identified in Bowles (2008) may apply: First, people could perceive the availability of costly insurance as a signal that 'buying' security is everyone's own responsibility (framing effect). Second, the fact that other people choose insurance might hint at their low commitment or trust in the existing solidarity transfer scheme and would provoke a negative response by reducing one's own pro-social giving (information effect for conditional co-operators). The crowding-out effect might also lead to individualization and the breaking apart of traditional structures which also affects other spheres of life.

There is already some literature specifically suggesting that insurance might crowd out solidarity transfers within the network. However this literature is either theoretical (Attanasio 
and Ríos-Rull 2000; Coate and Ravallion 1993) or is based on non-experimental data. In addition, as in (Dercon and Krishnan 2003; Jowett 2003), transfers are not measured directly. ${ }^{4}$ In observational studies it is also not possible to disentangle the processes leading to the crowding-out effect. Are the insured reducing their transfers or the uninsured? Which are the motives that drive peoples' decisions?

This paper delivers the first experimental evidence on whether informal solidarity is reduced by formal insurance in developing countries. Our design tries to reflect reality as much as possible. We model risk in a behavioral game using lotteries that involve rolling a dice. Every participant is provided with an initial endowment and depending on the dice roll she is allowed to keep all or part of it. Informal risk-sharing is implemented in nonanonymous groups of three broadly following the design of Selten and Ockenfels (1998). After the lottery is played, each group member can transfer some money to the other group members. Insurance is introduced via offering alternative lotteries that are safer but require some ex-ante fixed payment. We test two variations of insurance: one protects only against catastrophic shocks, while the other is more comprehensive type and covers also medium shocks.

Since acts of giving are not always fully voluntary but are "demanded" from the network members (Platteau 2000; Hoff and Sen 2006; Grimm et al. 2011; Comola and Fafchamps 2010), we implemented the possibility of secretly saving money in a "lockbox treatment" where players can pretend to have a medium shock (instead of no shock) and hide their money from the risk sharing group. This device relates to another aspect of microfinance: Resources allocated to (formal) saving products might be harder to monitor by the network (e.g. mobile banking) or might be regarded as non-liquid by the network. Formal saving thus may reduce informal risk-sharing. In fact, many people in developing countries are willing to pay considerable premia and even accept negative real interest rates in order to keep their money at a safe place such as formal banks, rotating savings and credit associations (ROSCAs) or deposit collectors. ${ }^{5}$ There is very little research on the effect of such saving

\footnotetext{
${ }^{4}$ Attanasio and Rios-Rull (2000) present an economic model explaining that under some conditions public insurance leads to a decrease in informal transfers. Dercon and Krishnan (2003) show that consumption is more responsive to shocks if there is food aid in rural Ethiopia, and Jowett (2003) find that there is less health insurance uptake if informal financial networks and social capital are stronger (or vice versa). This empirical evidence is in line with crowding out of private by public support schemes, however, both studies are based on non-experimental data and transfers are not measured directly, to mention only two weaknesses. The research is made more difficult by the fact that the measurement of informal solidarity and transfers is a difficult task. (Comola and Fafchamps 2010) for example use data where the receiver and sender both report on transfers. They show that the information from the two parties is largely inconsistent.

${ }^{5}$ In Africa, for example, especially women are willing to entrust their money with "Susu men" to withdraw it from their network (Besley 1995, p. 2150) or to put it in formal saving accounts with effectively negative interest rates (Dupas and Robinson 2009). There is also evidence that people prefer saving products that reduce their
} 
mechanisms on informal risk sharing. Especially research focusing on the 'hiding mechanism' (i.e. to save money in order to make it illiquid or invisible to others) is missing up to now. ${ }^{6}$

In contrast to real world data the controlled environment of our behavioral game allows monitoring the transfers and choices of participants perfectly and thus delivers much more reliable empirical results.

We find that secret savings that inhibit the observability of shocks reduces solidarity transfers substantially. People overwhelmingly use the option to hide money and then reduce their transfers. This is evidence for a strong role of extrinsic and for less intrinsic solidarity, as participants use the secret saving device most of the time and strongly decrease their willingness to transfer if it is available. Once the saving option is available, formal insurance does not lead to significant crowding-out effects on transfers, and formal insurance is now effective in significantly smoothing the loss distribution. The reason is that solidarity is already low when secret saving is possible such that additional crowding-out is limited. On the other hand, if participants cannot secretly save and solidarity is relatively high there is significant crowding-out. The positive effect of the insurance mechanism on the lower tail of the distribution is completely offset by the negative effect of decreased solidarity transfers in this case.

The fact that the crowding-out effect can completely offset the protection offered by the insurance hinges on the incomplete take-up. If everybody was insured nobody would be left with a catastrophic outcome even in the complete absence of solidarity transfers. Yet, while around half of all participants opt for insurance if they have the choice, there is a substantial part remaining uninsured. Those uninsured now face a much higher risk of being left alone with a bad outcome than in the scenario when nobody can be insured.

The main conclusions of the empirical analysis can be summarized succinctly as follows: In the absence of a secret savings option, substantial solidarity transfers are observed. These transfers must be motivated mostly by peer pressure rather than true altruism, because

\footnotetext{
liquidity even without an increase in interest rates. There exist Commitment Saving products, where people commit to save in an account and are not permitted to withdraw any money for a predefined time period or until a certain balance is reached. Ashraf, Karlan, and Yin (2006) found in a randomized experiment in the Philippines that $28,4 \%$ of existing or former bank clients opened a commitment savings account and saved significantly more within one year than people who opened a "normal" account.

${ }^{6}$ To the best of our knowledge there are only three papers (two unpublished) on the effect of savings on informal risk-sharing. Brune et al. (2011) show that a commitment saving product increases savings and decreases transfers to the social network. Flory (2011) analyzes a randomized field experiment on the effect of a mobile van offering saving services, amongst others, in India. Surprisingly, he finds a positive effect of savings on the incidence of gifts received by poor non-savers, but no effect on the amount. Chandrasekhar, Kinnan, and Larreguy (2010) conducted a behavioral field experiment in India to test the effect of saving, focusing on its inter-temporal income smoothing role. They find no effect of saving on risk-sharing. Yet, they make saving perfectly observable and only test its effect when risk sharing is already low due to a limited commitment treatment.
} 
most people immediately hide money if it becomes possible. If formal insurance is introduced, the voluntary transfers drop substantially and, depending on the price of the insurance, people are actually worse of. The introduction of formal insurance can even have persistent effects on crowding out voluntary transfers, even after insurance is removed. On the other hand, once people can hide money, the situation changes though. The reduced observability of shocks makes people unwilling to provide generous transfers, substantially increasing income risk. Formal insurance can now help to reduce risks and, in our simulations, can even offset the negative effects of the possibility of hiding money. Further simulations confirm that without the possibility to hide insurance would be more efficient when crowding out could be prevented. Yet, much larger decreases in vulnerability would be achieved with full insurance uptake.

These findings indicate that the effectiveness of formal insurance can depend on the availability or existence of other financial instruments: Formal insurance can be ineffective (e.g. when no saving device is available and solidarity is potentially high) or can be effective (e.g. when secret saving is possible and informal solidarity is limited). ${ }^{7}$ One lesson is to offer insurance if saving devices are in place or should be introduced (for reasons beyond the scope of our experiment). In fact, under these circumstances insurance decreases the risk of bad outcomes to levels without the saving device, thus exactly offsetting the breakdown of informal solidarity. On the other hand, when resources within the risk-sharing network can be closely monitored and solidarity is high introducing market-based insurance products with incomplete coverage of the whole population could have disappointing results - causing administrative costs without affecting overall vulnerability.

The remainder of the paper is organized as follows. Section II describes the experimental setup including treatments, hypotheses, implementation and subject pool. We discuss empirical results in sections III and IV and conclude in section V.

\footnotetext{
${ }^{7}$ Of course, there are also other reasons for low-solidarity that are outside of the scope of our experiment, e.g. high ethnic or linguistic fractionalization.
} 


\section{Setup of the experiment}

We model risk in a behavioral game using lotteries that involve rolling a dice. ${ }^{8}$ Every participant is provided an initial endowment of 200 Philippine Pesos (PhP) and depending on the dice roll she is allowed to keep all or part of it. ${ }^{9}$ This design reflects the risk to lose money instead of providing participants with the possibility of winning money. ${ }^{10}$ Informal risksharing is implemented in groups of three according to the standard solidarity game procedure (Selten and Ockenfels 1998). ${ }^{11}$ Contrary to most economic lab experiments we do not restrict our sample to students, nor do we make groups anonymous. The participants are rural villagers in the Philippines. We are convinced that this is more compatible with the idea of risk sharing at the village level and strengthens external validity of our results. After the lottery is played, the group is allowed to talk. Thereafter each member of the group can transfer some of his money to each of the other group members. Insurance is introduced via offering alternative lotteries that are safer but require some fixed payment ex ante.

\section{- Treatments -}

We test two variants of insurance, compared to no-insurance. One insurance protects against half of all loss types and is more expensive, while the other insurance covers half of catastrophic shocks only.

We first explain the no-insurance treatment, which we refer to as Option A. Every participant has an initial endowment of 200 Philippine Pesos. This is the amount paid out if the dice shows a 1,2 or 3 , i.e. which we label as "no shock" (no loss). If the dice shows a 4 or 5 a

\footnotetext{
${ }^{8}$ We benefited from the work of Barr and Genicot (2008) who combine a lottery choice with risk-sharing after the result is determined. They test different enforcement mechanisms in their experiment and find strong evidence for intrinsic motivation of giving, as substantial risk sharing takes place even if individuals can secretly opt out of the solidarity group. However, in their experimental procedure, lottery choice is not a treatment, so the effect of introducing insurance cannot be identified. Also, interpretation of the many gamble choices (according to Binswanger, 1980) as different insurance products is difficult. There are other experiments that come closer to our idea. Trhal and Radermacher (2009), for example, compare solidarity in treatments with and without gamble choice. Yet, the 'non-insurance' lotteries are not the same in both treatments and some other details do not fit our purpose. We consequently designed a novel behavioral experiment that is described in the following.

${ }^{9}$ The amounts where such that the expected payoff of participating in the experiment (237 PhP, or about 5-6 USD) equals about one day of minimum wage in the formal sector. The expected amount includes a show-up fee of $100 \mathrm{PhP}$ that every participant received for sure.

${ }^{10}$ Harrison and Rutström (2008) stress the importance of the reference point, referring to prospect theory (Kahneman and Tversky 1979) that allows subjective probability weighting, a reference point and different utility functions for losses and gains.

${ }^{11}$ There are problems with the 3-player approach since often winners do not anticipate that the other winner might also give. This leads to the strange situation that the player with the worst shock leaves the experiment with the highest earnings. However, this happened only eight out of 279 times. We also believe that two player relations are different from risk-sharing groups and thus not adequate for our experiment.
} 
medium shock occurs (losing half), and a 6 implies a catastrophic shock (losing almost everything). More precisely, if the medium shock occurs participants lose 100 of their initial 200 Pesos and if the catastrophic event occurs they lose 180. In case of no shock, participants do not lose any money, but keep all their 200 Pesos.

The two insurance variants are called option B and option C. ${ }^{12}$ For option B participants have to pay 45 Pesos in advance and half of all losses are covered. The price for option $\mathrm{C}$ is only 20 Pesos, but half of only the catastrophic loss is covered. (The prices 20 and 45 are chosen to reflect that more comprehensive schemes entail higher administrative costs. The more comprehensive insurance covers more shocks and is therefore confronted with more claims, and also higher administrative costs, which translates to lower expected payoffs.) Table 1 shows the payout for the no-insurance case (Option A) and the two insurance options B and C.

The advance cost of insurance thus is always the 'guaranteed loss' in case of no shock. In general, different options lead to a different spread of payoffs; the lower the standard deviation, the lower the expected total payoff. Option B is most costly, not only regarding absolute price but also when looking at the expected loss. Yet the risk, as represented by the standard deviation of the payoff, is smaller than for options A and C. Option C is an intermediate case with an interesting additional feature: Due to the low price and the focus on the catastrophic loss it can secure an even higher minimum payoff than option B. Because of this, individuals with minimax preferences would prefer $\mathrm{C}$ over $\mathrm{B}$. Both options $\mathrm{B}$ and $\mathrm{C}$ reflect typical insurance products where full coverage is impossible. ${ }^{13}$ With two insurance products, we are able to discuss demand for different insurance products and create a different take-up which might lead to more or less crowding-out. We are also able to detect product inherent effects that interact with solidarity. (If people had a constant relative risk aversion utility function $u(c)=\left(c^{1-\rho}\right) /(1-\rho)$ with risk parameter $\rho$, where $\rho>0$ for risk-averse individuals, in the absence of any solidarity transfers, all people with a risk-aversion parameter $\rho>0.34$ would buy insurance $C$ if available, whereas only people with $\rho>0.65$ would buy insurance B.

\footnotetext{
${ }^{12}$ We would have expected a higher crowding-out effect by labeling the lotteries as "insurance" instead of "option" but decided to leave this for future research.

${ }^{13}$ E.g. in most developing countries, health insurance covers only the medical expenses (often below $100 \%$ ), but not lost income due to lost labor, i.e. working time. The more comprehensive insurance could be like the state owned medical insurance scheme and the catastrophic insurance could be like different rainfall or crop insurance in the region.
} 
Table 1: Losses (in PhP) under different (insurance) options

\begin{tabular}{c|ccc|cc}
\hline \hline Dice Result & $\begin{array}{c}1,2,3: \\
\text { no } \\
\text { shock }\end{array}$ & $\begin{array}{c}4,5: \\
\text { medium } \\
\text { shock }\end{array}$ & $\begin{array}{c}\text { 6: } \\
\text { catastrophic } \\
\text { shock }\end{array}$ & $\begin{array}{c}\text { Expected } \\
\text { Loss }\end{array}$ & $\begin{array}{c}\text { Std-Deviation } \\
\text { of Loss }\end{array}$ \\
\hline Option A & $\mathbf{- 0}$ & $-\mathbf{1 0 0}$ & $-\mathbf{1 8 0}$ & -63.3 & 68.7 \\
Option B & $\mathbf{- 4 5}$ & $\mathbf{- 9 5}$ & $\mathbf{- 1 3 5}$ & -76.7 & 34.4 \\
Option C & $\mathbf{- 2 0}$ & $\mathbf{- 1 2 0}$ & $\mathbf{- 1 1 0}$ & -68.3 & 48.5 \\
\hline \hline
\end{tabular}

Note: The initial endowment is $200 \mathrm{PhP}$ in each round. The loss in case of "no shock" is the price of the insurance options participants have to pay upfront, i.e. $45 \mathrm{PhP}$ for option $\mathrm{B}$ and $20 \mathrm{PhP}$ for option $\mathrm{C}$.

In real life, observing what everybody gives to you is normally unproblematic, but perfectly observing individual shock levels of others is maybe not possible. Thus in some scenarios we did allow participants to pretend a medium shock. Catastrophic losses might on the other hand be observable to everybody. Therefore, observability of medium shocks was reduced in a secret saving treatment. ${ }^{14}$ If the dice result was 1,2 or 3 (no shock) individuals could decide to save the monetary difference to a medium shock in a secret lockbox. This information was private to the individual and group members were only informed about the amount the person had after the lottery/lockbox stage. Permitting saving in the lockbox thus made it impossible for the co-players to distinguish between no shock and a medium shock. The aim of this treatment is to increase external validity of our study and to show the effect of secret saving on solidarity, a potential side effect of (formal) saving products, e.g. mobile banking. It is still possible for people with no shock to help others in case of need, but a lot of solidarity based on peer pressure will be reduced.

To test the effects of the two insurance types and of the secret saving device, the behavioral experiments were implemented as outlined in Table 2. In six villages (treatment block A) no insurance is offered in rounds one and two. Hence, participants have no choice and always play option A. In round three both insurance types are introduced and participants can choose between all three options. In eight villages (treatment block $\mathrm{AB}$ ), insurance option $\mathrm{B}$ is offered in round one, no insurance in round two and again insurance option $\mathrm{B}$ in round three. In another eight villages (treatment block AC), the same is done with option C. In order

\footnotetext{
${ }^{14}$ To not influence participants we did not call this 'saving', but rather explained the possibility to "put money in a lockbox" and the related mechanics.
} 
to test the main and interaction effects of the secret saving possibility, the secret saving device was implemented in the first two rounds in half of all villages. ${ }^{15}$

It is important to note that each group plays all three rounds, but that for only one of these three rounds a real payout takes place. The round that is being paid out is randomly chosen after all three rounds have been completed. The participants knew this in advance. Hence, apart from possible learning effects, no dynamic, strategic or endgame effects can occur.

Table 2: Treatment plan for insurance types

\begin{tabular}{|c|c|c|c|}
\hline & $\begin{array}{c}\text { Block } A \\
\text { (6 villages) }\end{array}$ & $\begin{array}{c}\text { Block AB } \\
\text { ( } 8 \text { villages) }\end{array}$ & $\begin{array}{c}\text { Block AC } \\
\text { ( } 8 \text { villages) }\end{array}$ \\
\hline \multirow[t]{2}{*}{ Round 1} & Option A & $\frac{\text { Choice: }}{\text { Option A or B }}$ & $\frac{\text { Choice: }}{\text { Option A or C }}$ \\
\hline & 3 save, 3 no save & 4 save, 4 no save & 4 save, 4 no save \\
\hline \multirow{2}{*}{ Round 2} & Option A & Option A & Option A \\
\hline & 3 save, 3 no save & 4 save, 4 no save & 4 save, 4 no save \\
\hline \multirow[t]{2}{*}{ Round 3} & $\frac{\text { Choice: }}{\text { Option A, B or C }}$ & $\frac{\text { Choice: }}{\text { Option A or B }}$ & $\frac{\text { Choice: }}{\text { Option A or C }}$ \\
\hline & 3 save, 3 no save & 8 no save & 8 no save \\
\hline
\end{tabular}

Note: In each block, in half of the villages the games were played with the "secret-saving device" and in the other half without. This is indicated by " 3 save, 3 no save" or " 4 save, 4 no save". The notion " 8 no save" means that in Round 3 the secret-saving device was not available anymore.

Our main hypotheses formulated prior to conducting the experiment are:

(I) Solidarity transfers are reduced by the availability of insurance.

(II) There is a persistent reduction of solidarity even if insurance is removed.

(III) Solidarity transfers are reduced by the possibility of secret saving

The effect of the different insurance types can be tested by comparing treatment block $\mathrm{A}$ versus block $A B$ versus block $A C$ in the first round (Hypothesis I). Treatment A serves as a control here. The persistence effect of access to insurance on solidarity can be tested in the second round (Hypothesis II), comparing it to the first round. The effect of secret saving can be assessed by comparing the 11 villages, where secret saving was made possible, to the other half (Hypothesis III). In addition to these three hypotheses the experiment permits the analysis of many more aspects. For example, the third round allows for a comparison of demand for

\footnotetext{
${ }^{15}$ In the third round it was removed again in most cases. We intended to analyze persistence in its effect by this design choice, but we do not find any statistically significant evidence.
} 
the insurance variants while competing in one market in block A. This is the only scenario where participants can choose between two insurance options. In the other scenarios only insurance versus no-insurance is available. With the simultaneous availability of two insurance options we can examine how individuals choose from a menu of insurance options. Additionally, it is possible to compare take-up of option B and option C on separate markets with limited ability to observe shocks (secret saving). ${ }^{16}$ Furthermore, the third round also delivers more observations for the pooled regressions at a later stage.

As an additional treatment, different network strengths of the player groups are examined. The groups of player were formed in two ways: As a standard procedure a randomly selected person had to invite two other household heads that he knows very well to join the experiment. In half of the villages these players would form a group. We will refer to them as endogenous groups or as strong networks. In the other half of the villages, however, we mixed up groups, and participants would play with two random partners from their village. ${ }^{17}$ These groups will be called exogenous or random or "weaker networks". The analysis of this additional covariate (network strength) is not the core of this paper, and it is impossible that it causes a bias of the main treatment effects because it is balanced across insurance treatment blocks. However, we will control for it in regressions since it is not exactly balanced in each subcell (e.g. in Block A there are only 3 villages with saving and 3 without saving) and because there might be interesting interactions between network strength and the main treatments.

\section{- Implementation -}

All participants were assigned to groups of three and received player numbers upon arrival. The composition of groups was done in two ways, as explained above: In half of the villages, they would remain in their self-selected groups of three, i.e. they had registered themselves together with two friends. In the other half of the villages, they were randomly re-assigned to two new co-players. To indicate the group-allocation-scheme, we will later use a dummy variable labeled "weaker network", where weaker network takes the value 0 in the former

\footnotetext{
${ }^{16}$ In treatment blocks AB and AC we varied the ability to observe shocks (labeled as "secret saving") in some of the villages to test more hypotheses, e.g. persistence of the secret saving device effect.

${ }^{17}$ The differences between the two types of groups can be found in participants' self-assessed relation to their group members. While more than $55 \%$ described their co-players as "close family" in endogenous groups, less than $30 \%$ do so in exogenous groups. Even though we do not believe that participants were very accurate in their classification (30\% seems very high for groups formed at random), the differences between the two types of groups is considerable.
} 
villages and the value 1 in the latter villages. This is to indicate that in the former villages, the groups were self-selected whereas in the latter villages, the groups were formed at random.

The groups stayed together for all three rounds, and people in a group knew the other two members. After answering the pre-questionnaire, participants were seated to receive the introduction to the game. In an effort to make the rounds as independent from each other as possible, we made sure that signaling, punishment and the like cannot take place. Therefore decisions of co-players were never revealed and we did not allow for communication after the transfer choice. Group members were even seated separately to inhibit communication and no group was ever without supervision of at least one assistant. The instructor pointed out that communication within groups is forbidden outside the communication stage, that violations of the treatment protocol will lead to the exclusion from the experiment, that three games will be played independently from each other and that only one of them will be paid out at random.

The complete experimental procedure of one round is summarized in Figure 1. First, the instructor explains the game to all participants jointly, and everybody receives a plastic envelope with graphical instructions for this round and their initial endowment of $200 \mathrm{PhP}$ in toy money. Before participants go to private room 1, they answer a set of questions in order to test their understanding of the game. ${ }^{18}$ If the current round permits insurance options (see Table 2), participants are given a choice of lotteries. Otherwise only the standard lottery (Option A) is available. ${ }^{19}$ After the participants make their lottery choice and pay the related price, they roll a dice to determine the loss. Where secret saving is available, players with no shock could then decide to hide a fixed amount of their money or not. After all have chosen whether to hide or not, the members are allowed to talk for approximately five minutes, before each individual separately goes to another private room 2 . At this point, the amount that the two co-players took out of the first private room is revealed (endowment, minus insurance premium, minus loss due to shock, minus secret saving). Importantly, only the net payout is revealed, and not whether insurance has been bought, or whether shocks took place or whether secret savings have been made. From these payouts, however, one can induce who has purchased insurance and who did not. The participant then decides about transfers, i.e. if and how much to give to each of the co-players. Everybody is completely free in the way he or she shares the money. These transfers are never revealed to anyone. Only after all three

\footnotetext{
${ }^{18}$ The test questions can be found in the appendix. When participants made mistakes, the research assistants explained the setup once more. Only those who finally answered all questions correctly were allowed to participate, but fortunately we only had to exclude few participants.

${ }^{19}$ Option A is not framed as the default option, but lotteries are instead assigned neutral names: Angola (A), Botswana (B) and Cameroon (C). However, participants knew that one option is for free, while potential alternatives would require an ex-ante payment from the initial endowment.
} 
rounds have been completed and after one round has randomly been chosen for pay-out, do the players receive any feedback: They receive cash in hand and from the received cash they can partly deduce whether they have received any transfers, but without knowing from whom. Hence, transfers from the past cannot affect the behavior in future rounds.

Figure 1: Experimental Procedure

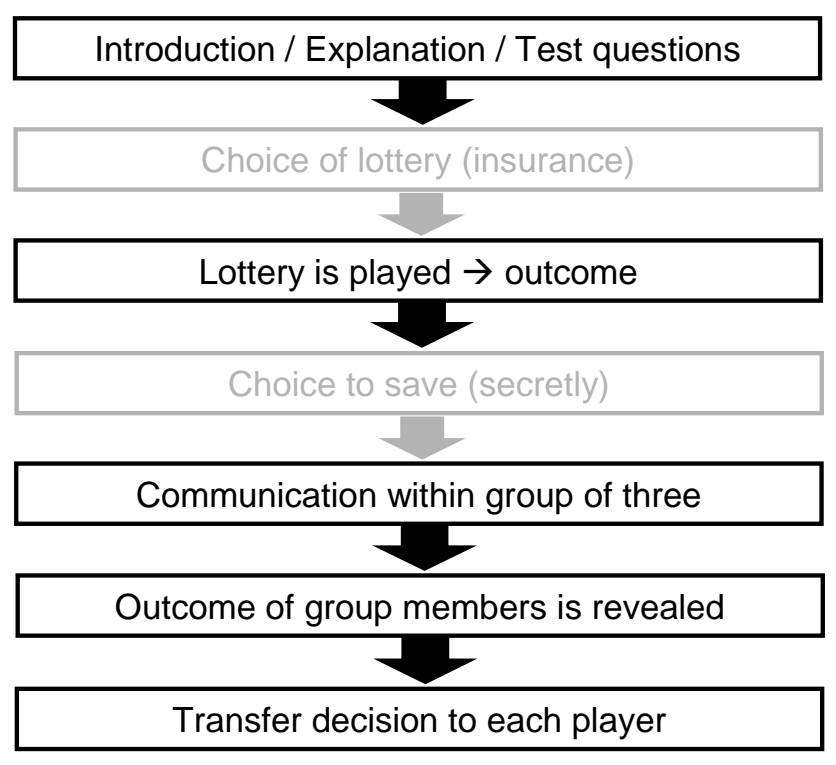

private room 1

private room 1

private room 1

private room 2

private room 2

To ensure that experimental conditions did not change, the same team of ten assistants was employed for the same job all the time, strictly adhering to the experimental protocol (i.e. the same person always read the protocol, the same assistants were sitting in room 1 and room 2 etc). In both private rooms, decisions were recorded by the research team. Communication within a group was restricted to the communication stage. Whenever there was an unclear situation, the researcher was present to decide on the issue. After all three rounds had been played, and after the completion of the post-game questionnaire and the random determination of which round to be paid out, the participants were handed out their winnings in private. All participants received a fixed show-up fee of $100 \mathrm{PhP}$ in addition to their payoff from the relevant round.

\section{- Subject pool -}

The experiment was conducted in the Western Visayas (Region VI), in the province of Iloilo. Existing databases suggest that the region is a slightly disadvantaged but not unrepresentative 
region within the Philippines. ${ }^{20}$ A two-stage random sampling procedure was applied throughout. First, we randomly determined the experimental sites, and then we drew participants within the selected barangay (lowest administrative level on the Philippines and often comparable to a village regarding size and structure). The exact combination of treatments played in one location according to the treatment plan was also determined randomly, but the randomization had to pass a balancing test regarding village size across the treatments.

The target population consists of low-income households in rural or partially urban areas. We therefore drew a random sample of 22 barangays whereby municipalities from the first income class (high income) and urban locations were excluded from the sampling process. ${ }^{21}$ Also very small (population below 500) and very big (population higher than 3000 ) barangays were not considered to make the sample more homogenous. ${ }^{22}$ Permission of the Punong Barangay (elected village representative) to conduct the research was obtained in all but one barangay, leading to its replacement by another random site. We made all possible efforts to visit also remote locations, and all 22 locations of the sample could finally be reached.

In the second sampling stage, the households were randomly chosen within a barangay. Our recruiters went to the location some days prior to the experiment, asked the barangay officials for permission to run the experiment, ensured the availability of facilities for the games and requested a list of households from which eight households were randomly selected. $^{23}$ The recruiters then noted the names of the eight households and handed out invitation letters to them. Only the household head or the spouse of a household - in special cases also adult children still living in the household - were allowed to take part in the game. We also checked with the Punong Barangay whether the invited household representatives are too old to participate. ${ }^{24}$ Each invitation had two additional invitation letters attached as well as the instruction to invite representatives from two more distinct households by choice. The sample size varied from 15 to 24 per village. The total number of observations is 466 .

\footnotetext{
${ }^{20}$ The Demographic and Health Survey 2008 (http://www.measuredhs.com/data/dataset/Philippines StandardDHS 2008.cfm) and a household survey conducted by the University of Mannheim in 2009 suggest the following: educational attainment is slightly below national average, poverty is higher and coverage with public health insurance is around average.

${ }^{21}$ Income Classification based on Department of Finance Department Order No.20-05 Effective July 29, 2005 (source: http://www.nscb.gov.ph).

${ }^{22}$ Four of the 22 barangay were already chosen at random for an earlier household survey. To link the data from both studies they were included even though one barangay was slightly too small (350) and another one slightly too large (3123).

${ }^{23}$ Every barangay was able to provide a complete household list.

${ }^{24}$ Our preferred age was between 18-60 years, but we mainly relied on the judgment of the Punong Barangay regarding the fitness of participants. Participants above age 70 are not considered, though.
} 


\begin{tabular}{|c|c|c|c|c|c|c|c|}
\hline \multirow[b]{2}{*}{ Variable } & \multicolumn{4}{|c|}{$\begin{array}{c}\text { All } \\
(\mathrm{N}=466)\end{array}$} & \multirow{2}{*}{$\begin{array}{c}\text { Block A } \\
(\mathrm{N}=132) \\
\text { Mean }\end{array}$} & \multirow{2}{*}{$\begin{array}{c}\text { Block AB } \\
(\mathrm{N}=167) \\
\text { Mean }\end{array}$} & \multirow{2}{*}{$\begin{array}{c}\text { Block AC } \\
(\mathrm{N}=167) \\
\text { Mean }\end{array}$} \\
\hline & Mean & Std. & Min & $\operatorname{Max}$ & & & \\
\hline Male & 0.31 & & 0 & 1 & 0.30 & 0.29 & 0.35 \\
\hline Household head & 0.31 & & 0 & 1 & 0.24 & 0.30 & $0.37^{\star \star}$ \\
\hline Married & 0.81 & & 0 & 1 & 0.83 & 0.80 & 0.80 \\
\hline Highest education: high school & 0.44 & & 0 & 1 & 0.49 & 0.48 & $0.37^{\star}$ \\
\hline Highest education: college or above & 0.25 & & 0 & 1 & 0.23 & 0.30 & 0.21 \\
\hline Age (in years) & 42.7 & 12.13 & 18 & 69 & 42.7 & 41.2 & 44.2 \\
\hline Regular monetary income? (dummy) & 0.23 & & 0 & 1 & 0.23 & 0.25 & 0.22 \\
\hline Skipped meals in Household last month & 0.30 & & 0 & 1 & 0.30 & 0.23 & 0.35 \\
\hline In debt with more than 1000 Pesos? & 0.57 & & 0 & 1 & 0.55 & 0.64 & 0.51 \\
\hline
\end{tabular}

Stars indicate significance level of Wilcoxon ranksum test for differences to mean in treatment block A. (See Table 2 for definition of the treatment blocks.) $* * * \mathrm{p}<0.01, * * \mathrm{p}<0.05, * \mathrm{p}<0.1$

Descriptive statistics of the participants are presented in Table 3. Most of them are female (69\%), and therefore the share of household heads is only $31 \%$. Educational level is relatively high with more than two thirds having attended at least high school (44\% stopped at this level and an additional 25\% reached college). Below 18 year olds were not allowed to take part in the game and individuals with 70 years and above are excluded from the analysis. Regarding the financial situation of households, less than a quarter report regular monetary income. Also, in $30 \%$ of households members had to reduce meals for financial reasons in the last month, which serves as a rough measure of poverty. $57 \%$ are in debt with more than 1000 Pesos, the equivalent of roughly 22 US dollars. ${ }^{25}$

Due to the randomized assignment to treatments, in expectation all characteristics are balanced, nevertheless in small samples some correlation remains. For example there is a higher share of household heads in treatment AC than in the control A and the educational attainment is slightly lower. The same is true for village characteristics, shown in Table A1 of the appendix. Especially income class of the municipality is somewhat different by chance across treatment blocks. Otherwise most characteristics are balanced. Nevertheless, the smallsample correlation in some characteristics hints at the importance of controlling for covariates in regressions.

\footnotetext{
${ }^{25}$ Around half of them owe the money to friends or relatives.
} 


\section{Empirical Analysis}

In the following, we will first consider some descriptive results on the effect of insurance and secret savings using the comparisons implied by the treatment plan (see Table 2). Afterwards we will control for different shock distributions ${ }^{26}$ across treatments via matching, before employing a parametric regression model to control for more possibly imbalanced covariates and to gain further insights. Using the regression results we simulate loss/payout distributions under different insurance and saving regimes.

\section{- Descriptive results -}

A first finding is that the safer lottery options are frequently demanded by participants: On average $46 \%$ 'buy' insurance if they have the possibility to do so. Figure 2 illustrates the demand in treatment blocks $\mathrm{AB}$ and $\mathrm{AC}$ by round, and shows that lottery type $\mathrm{C}$ is more popular than type B, especially in the last round. It is interesting to note that the demand for insurance $\mathrm{C}$ increases from Round 1 to 3 , whereas the demand for insurance $\mathrm{B}$ decreases. Since the participants did not receive any information or feedback about received transfers during the game, their change in behavior can only be explained by imitating others' choices or by learning from experienced shocks. It appears that while buyers of insurance $\mathrm{C}$ where happy with what they bought (insurance against catastrophic losses), for many buyers of insurance B the product might have been too expensive. This difference in 'client satisfaction' is reflected in different retention rates from round 1 to 3 . While $72 \%$ of the insured with type $\mathrm{C}$ in the first round chose insurance in round three again, only $57 \%$ renewed their insurance B.

Figure 2 only shows take-up for treatment blocks $\mathrm{AB}$ and $\mathrm{AC}$, where either only option $\mathrm{B}$ or only option $\mathrm{C}$ are offered. In treatment block $\mathrm{A}$ in round three, both insurance options B and C are available at the same time. There $43.4 \%$ choose option $\mathrm{C}$ and $17.8 \%$ choose option B (while the remaining $40 \%$ choose the no-insurance option A).

The characteristics of the insured versus non-insured by insurance treatment block (again using block $\mathrm{AB}$ and $\mathrm{AC}$ ) in rounds one and three can be found in Appendix I (tables A3 and A4). While no clear picture emerges across all comparisons the poor seem to have a slightly higher tendency to take up, i.e. the insured tend to be more indebted and had to skip

\footnotetext{
${ }^{26}$ By the nature of the experiment, the shock distributions would be identical across treatments if sample size was sufficiently large. But given our small sample size, imbalances occur.
} 
meals more often. This suggests that low-income participants have more need for money from the experiment and behave more risk averse.

Figure 2: Demand for insurance on separate markets

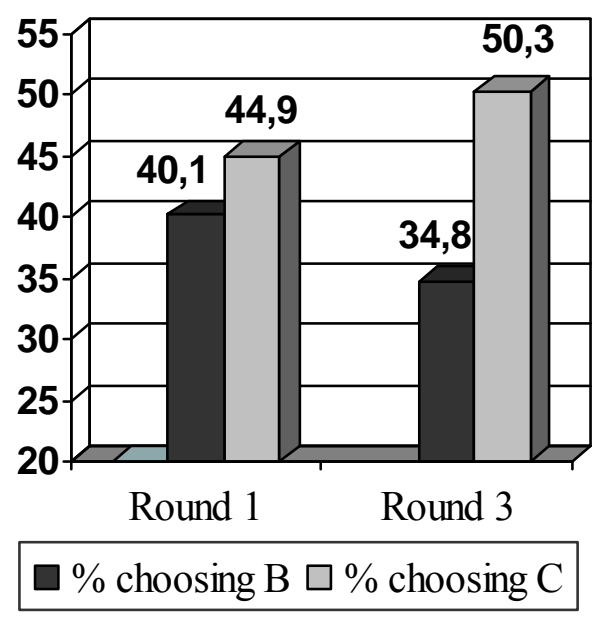

Note: only treatment blocks $\mathrm{AB}$ and $\mathrm{AC}$ (without block $\mathrm{A}$, round 3)

Before we examine transfers in the following, we note that "secret saving" was used overwhelmingly: Whenever secret saving was possible, it was used in $94 \%$ of all cases. This overwhelming use to hide money leads us to believe that this also reflects people's behavior in real life. ${ }^{27}$

Transfers between group members vary greatly between 0 and 100, with a mean of 10.6 pesos. Figure 3 displays the distribution of the 2730 observed payments from sender to recipient. ${ }^{28}$ In $57 \%$ of all cases transfers are zero. ${ }^{29}$ The standard deviation is 16.5 pesos and the mean is 10.6 pesos.

\footnotetext{
${ }^{27}$ Remember that participants can only save and thus pretend a medium shock if they have no shock.

${ }^{28}$ Each participant of the 466 makes two transfer decisions per round. However, one group dropped out in round two and another group in round three, because at least one player could not continue the game due to sickness or personal reasons. Also transfers from and to participants older than 69 are excluded. Note that the distribution function in Figure 3 is non-smooth since transfers were only possible as multiples of five, i.e. 0, 5, 10, 15... We used toy money where the smallest note was 5 Peso.

${ }^{29} 43 \%$ of transfers are zero if the sender is better off than the recipient.
} 
Figure 3: Cumulative distribution and histogram of transfers
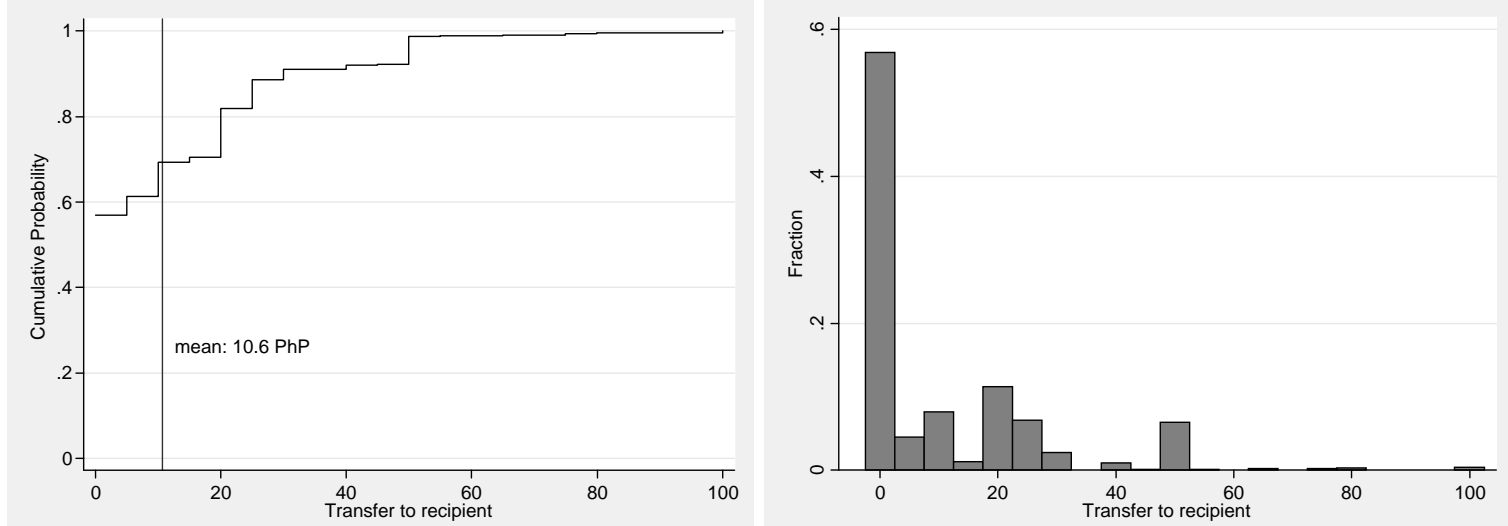

The transfers are described in a compact form in figure 3; however they do not necessarily indicate effective redistribution of the money, as every group member can transfer to the other and vice versa. Let $T_{i j}$ be the transfers from player $\mathrm{i}$ to $\mathrm{j}$. Real redistribution is the result of net transfers $T_{i j}-T_{j i}$, that is transfers from player $\mathrm{i}$ to $\mathrm{j}$ minus transfers from $\mathrm{j}$ to $\mathrm{i}$. Therefore it will not be sufficient to compare average transfers across treatments, as they might simply reflect a different inclination to give in general, which is completely irrelevant for redistribution. ${ }^{30}$ Solidarity works (in the sense of risk sharing) if the better-off give more to the worse-off than the other way around. For the descriptive analysis of the treatment effects we will therefore start with a comparison of net transfers from those with a zero or less severe shock to those with a more severe shock. This means we only look at net redistribution from those without a shock to co-players with at least a medium shock, and of those with a medium shock to co-players with a catastrophic shock. We refer to these as "net transfer to disadvantaged co-player".

Table 4 shows the average net transfers to the disadvantaged co-player by round and treatment block. Net transfers in treatment blocks $\mathrm{AB}$ and $\mathrm{AC}$ are shown relative to block $\mathrm{A}$. Net transfers in the secret saving treatment are shown relative to the no saving case. Remember that the comparison in the first round allows testing the effect of different insurance types by comparing treatment block $\mathrm{A}$ with block $\mathrm{AB}$ and with block $\mathrm{AC}$ in the first round (Hypothesis I). Treatment A serves as a control. (Note that in these cases participants never could choose between insurance B and C. They could only choose between one

\footnotetext{
${ }^{30}$ Imagine a treatment that leads all participants to give more. If this effect is the same for the better- and the worse-off, the two level effects will just cancel out after mutual transfers, and redistribution is unaffected.
} 
insurance type versus option A.) One should also keep in mind that these comparisons across treatment blocks give the effect of insurance availability, not of take-up itself. ${ }^{31}$

The persistent effect of insurance on solidarity can be tested in the second round (Hypothesis II). In round one, insurance was available in the blocks AB and AC. However, in the second round, insurance was not available anywhere. Hence, one should not see any difference in transfers between blocks $\mathrm{A}, \mathrm{AB}$ and $\mathrm{AC}$, unless the availability of insurance in Round 1 had a persistent effect. Finally, we can also estimate the effect of the secret saving device by comparing saving with no saving treatment in both rounds (Hypothesis III).

Table 4: Net transfers to disadvantaged co-players

\begin{tabular}{|c|c|c|c|c|c|c|c|c|c|c|}
\hline & & $\begin{array}{l}\text { All } \\
\text { (1) }\end{array}$ & $\begin{array}{c}\text { BlockA } \\
\text { (2) }\end{array}$ & $\begin{array}{c}\text { BlockAB } \\
\text { (3) }\end{array}$ & $\begin{array}{c}\text { BlockAC } \\
\text { (4) }\end{array}$ & $\begin{array}{c}\text { Block } \\
\text { AB vs. A } \\
\text { (5) }\end{array}$ & $\begin{array}{c}\text { Block } \\
\text { AC vs. A } \\
\text { (6) }\end{array}$ & $\begin{array}{l}\text { No } \\
\text { saving } \\
(7)\end{array}$ & $\begin{array}{c}\text { Saving } \\
\text { (8) }\end{array}$ & $\begin{array}{c}\text { Saving vs. } \\
\text { no saving } \\
\text { (9) }\end{array}$ \\
\hline Round & Variable & Mean (Std) & Mean & Mean & Mean & Difference & Difference & Mean & Mean & Difference \\
\hline \multirow[t]{2}{*}{1} & $\begin{array}{l}\text { Net Transfer } \\
\text { to recipient }\end{array}$ & & 10.9 & 9.8 & 5.7 & -1.1 & $-5.2^{*}$ & 12.2 & 4.7 & $-7.5^{\star *}$ \\
\hline & No. obs & 270 & 68 & 107 & 95 & 175 & 163 & 141 & 129 & 270 \\
\hline \multirow[t]{2}{*}{2} & $\begin{array}{c}\text { Net Transfer } \\
\text { to recipient }\end{array}$ & $15.1(23.5)$ & 16.1 & 18.3 & 11.7 & +2.2 & -4.4 & 19.9 & 10.4 & $-9.5^{\star \star \star}$ \\
\hline & No. obs & 282 & 69 & 101 & 112 & 170 & 181 & 140 & 142 & 282 \\
\hline
\end{tabular}

Stars indicate significance level of Wilcoxon ranksum test for differences to mean in treatment $\mathrm{A}$ $* * * \mathrm{p}<0.01, * * \mathrm{p}<0.05, * \mathrm{p}<0.1$

On average, participants redistribute 8.6 pesos in the first round and 15.1 pesos in the second round from the better-off to the worse-off. Solidarity thus seems to work in tendency, although transfers have large standard deviations.

In columns (2) to (6) we examine how transfer behavior changes when insurance is available. Columns (2) to (3) give the averages in each treatment block, and columns (4) and (5) show the differences to Block A, augmented by a Wilcoxon ranksum test. We see that the availability of option $\mathrm{C}$ decreases transfers by 5.2 in round 1 and also in round 2. (These results are rather noisy, and will be examined further below.) For insurance option B the results are less clear.

In columns (7) to (9) we examine the effects of the possibility of secret saving. The option of secret saving has a very strong and significant negative effect on solidarity in both

\footnotetext{
${ }^{31}$ The third round is not shown in Table 4 as it is less comparable: First, there is no control group, because both insurance types are available in the third round of treatment block A. Second, the comparisons are not balanced, as observability of shocks (secret saving device) was changed in the third round of treatment blocks $\mathrm{AB}$ and $\mathrm{AC}$ (see treatment plan in Figure 2). Hence, simple descriptive statistics would not be meaningfully comparable for round 3. (Round 3 will be used in regression analysis later.)
} 
rounds. We also had noted before that almost all people (94\%) make use of the secret saving option when possible. This rather strong finding indicates that if people know that they themselves as well as others can hide some resources, net transfers drop by around two thirds in round 1 and around half in round 2. These estimates are significant at the 5\% and $1 \%$ level, respectively.

In the following, we examine the interaction effects between secret savings and insurance availability. In Table 5 we compare across treatment blocks, using only those sessions where secret saving was not possible. We thereby estimate the effects of insurance availability, conditional on no possibility of secret saving. Hence, shocks are fully observable to other participants. Now we find that the effects of insurance become much more pronounced. Table 5 shows that the effects become larger and more significant. Here both treatment effects are significant at the 5\% (AB) and 10\% (AC) level, respectively, and have about the same size. Results suggest that net redistribution with insurance is only around half of what it would be without formal insurance.

We also find a persistent effect of insurance into the second round. In the second round, no insurance is available anywhere and any differences in mean transfers can only be due to persistent effects (Hypothesis II). Here we find that availability of insurance C displays a marginally significant persistent effect in the second round. This is not the case for the more comprehensive type $\mathrm{B}$, though. While we do not have conclusive evidence to explain this, we consider the framing effect (availability of insurance signals that participants are no longer responsible for smoothing shocks) to be more persistent for insurance type $\mathrm{C}$, as it is much cheaper and thus might be considered affordable to all. A more detailed discussion on this is given in section $\mathrm{V}$.

Table 5: Net transfers to disadvantaged co-players (Only sessions where saving was not possible)

\begin{tabular}{c|c|c|ccc|cc}
\hline \multirow{2}{*}{ Round } & \multicolumn{6}{c}{ No saving subsample } \\
\cline { 3 - 8 } & Variable & Mean (Std) & Mean & Mean & Mean & $\begin{array}{c}\text { Block } \\
\text { AB vs. A } \\
\text { Difference }\end{array}$ & $\begin{array}{c}\text { Block } \\
\text { AC vs. A } \\
\text { Difference }\end{array}$ \\
\hline \multirow{2}{*}{1} & $\begin{array}{c}\text { Net Transfer } \\
\text { to recipient } \\
\text { No. obs }\end{array}$ & $12.2(21.2)$ & 18.4 & 10.4 & 9.1 & $\mathbf{- 8 . 0 ^ { * * }}$ & $\mathbf{- 9 . 3 ^ { * }}$ \\
\hline \multirow{2}{*}{2} & $\begin{array}{c}\text { Net Transfer } \\
\text { to recipient } \\
\text { No. obs }\end{array}$ & 141 & 40 & 52 & 49 & 92 & 89 \\
\hline \hline
\end{tabular}

Stars indicate significance level of Wilcoxon ranksum test for differences to mean in treatment $\mathrm{A}$ $* * * \mathrm{p}<0.01, * * \mathrm{p}<0.05, * \mathrm{p}<0.1$ 
Table 6 shows the results when using only villages where secret saving was possible. Here we do not find any statistically significant differences. Also note that the difference between treatment blocks $\mathrm{A}$ and $\mathrm{AB}$ in the first round -9.0 albeit not significant - might well be explained by the lower amount that can be secretly saved with insurance $\mathrm{B} .{ }^{32}$ In general it is perhaps not surprising that we cannot observe further crowding-out if net transfers are already dramatically lower on average in the subset with the secret saving device (4.7 versus 12.2 $\mathrm{PhP}$ in round one, 10.4 versus 19.9 $\mathrm{PhP}$ in round two).

Table 6: Net transfers to disadvantaged co-players (Only sessions with saving)

\begin{tabular}{|c|c|c|c|c|c|c|c|}
\hline \multirow[b]{3}{*}{ Round } & \multirow[b]{3}{*}{ Variable } & \multicolumn{6}{|c|}{ Saving subsample } \\
\hline & & All & BlockA & BlockAB & BlockAC & $\begin{array}{c}\text { Block } \\
\text { AB vs. A }\end{array}$ & $\begin{array}{c}\text { Block } \\
\text { AC vs. A }\end{array}$ \\
\hline & & Mean (Std) & Mean & Mean & Mean & Difference & Difference \\
\hline \multirow{2}{*}{1} & $\begin{array}{l}\text { Net Transfer } \\
\text { to recipient }\end{array}$ & $4.7(19.7)$ & 0.2 & 9.2 & 2.1 & +9.0 & +1.9 \\
\hline & No. obs & 129 & 28 & 55 & 46 & 83 & 74 \\
\hline \multirow{2}{*}{2} & $\begin{array}{l}\text { Net Transfer } \\
\text { to recipient }\end{array}$ & $10.4(22.6)$ & 7.7 & 13.4 & 9.1 & +5.8 & +1.5 \\
\hline & No. obs & 142 & 32 & 54 & 56 & 86 & 88 \\
\hline
\end{tabular}

Stars indicate significance level of Wilcoxon ranksum test for differences to mean in treatment $\mathrm{A}$ $* * * \mathrm{p}<0.01, * * \mathrm{p}<0.05, * \mathrm{p}<0.1$

When interpreting these descriptive results, we need to keep in mind that the amount of redistribution is likely to depend on the degree of inequality in the group. For large sample sizes, the distributions of the dice-rolling-results would be equal across treatment blocks and rounds. For our small samples, though, the shock distributions implied by the dice rolling results are not exactly balanced. To deal with this issue, we proceed in two ways. First, we examine in the following nonparametric matching estimates, where transfers are only compared for identical shock situations. Second, we will thereafter use parametric regression models to control for the shock distribution and also other covariates. ${ }^{33}$

\footnotetext{
${ }^{32}$ Remember that to guarantee non-observability participants can always save the difference to the medium shock. This difference is lower (50 instead of $100 \mathrm{PhP})$ if participants are insured against half of the medium loss (with insurance type B). This fact is an additional reason to control for the amount saved in regressions.

${ }^{33}$ Comparing the shock dispersion across treatments and rounds does indeed reveal differences in the shock dispersion that are significant at the $10 \%$ level in some cases. As this is a result of dice rolls, it is by definition pure chance and large differences should never be present in large samples. However, in our case this is a smallsample correlation that might nevertheless bias results.
} 


\section{- Matching estimation results -}

A nonparametric way to control for different shock distributions across blocks is via exact matching. We do this separately for comparing block $\mathrm{AB}$ versus $\mathrm{A}$, and for comparing block $\mathrm{AC}$ versus A, and separately for round one and two, respectively. For every sender and recipient pair in treatment block $\mathrm{AB}$ with a certain shock combination, we look for a sender and recipient pair in treatment block A (control) with exactly the same shock combination. In addition, we also require that the shock of the third group member is also identical. We furthermore also require that the round number, the network strength and the availability of the saving device are the same. We test the effect of saving analogously, simply making secret saving the treatment variable and adding insurance availability and type as a control variable. Table 7 shows the average treatment effect (ATE) of the two insurance treatment blocks and the secret saving option using exact matching, separately for round one and two. The last two columns of Table 7 repeat the insurance treatment matching for the subset of villages without the secret saving possibility.

The results of Table 7 strongly confirm the results of the previous tables, often with higher significance levels. Availability of insurance type $\mathrm{C}$ is associated with lower solidarity transfers from the better to the worse-off. When restricting attention to villages without the secret saving lockbox we see larger effects, and also the persistent effect of insurance type C is significant (at the 10\% level). Effects are insignificant for the more comprehensive scheme B. Matching for the set of villages with a secret saving lockbox again does not show any significant effects. Effects of the secret saving device are negative, large and highly significant in both rounds.

Table 7: Average treatment effect (ATE) of treatment blocks on net transfers to disadvantaged co-players (with and without saving)

\begin{tabular}{|c|c|c|c|c|c|c|c|c|}
\hline & & \multicolumn{3}{|c|}{ all } & \multicolumn{2}{|c|}{ no saving } & \multicolumn{2}{|c|}{ saving } \\
\hline Round & Outcome variable & $\begin{array}{c}\text { Block } \\
\text { AB vs. A } \\
\text { (ATE) }\end{array}$ & $\begin{array}{c}\text { Block } \\
\text { AC vs. A } \\
\text { (ATE) }\end{array}$ & $\begin{array}{c}\text { Saving vs. } \\
\text { no saving } \\
\text { (ATE) }\end{array}$ & $\begin{array}{c}\text { Block } \\
\text { AB vs. A } \\
\text { (ATE) }\end{array}$ & $\begin{array}{c}\text { Block } \\
\text { AC vs. A } \\
\text { (ATE) }\end{array}$ & $\begin{array}{c}\text { Block } \\
\text { AB vs. A } \\
\text { (ATE) }\end{array}$ & $\begin{array}{c}\text { Block } \\
\text { AC vs. A } \\
\text { (ATE) }\end{array}$ \\
\hline 1 & $\begin{array}{c}\text { Net Transfer to recipient } \\
\text { Obs (On/off support) }\end{array}$ & $\begin{array}{c}-0.4 \\
131 / 44\end{array}$ & $\begin{array}{c}-6.9^{*} \\
140 / 23\end{array}$ & $\begin{array}{l}\mathbf{- 8 . 5 ^ { * * * }} \\
217 / 53\end{array}$ & $\begin{array}{c}-6.0 \\
75 / 17\end{array}$ & $\begin{array}{l}-10.3^{\text {** }} \\
82 / 7\end{array}$ & $\begin{array}{c}+7.1 \\
56 / 27\end{array}$ & $\begin{array}{c}-2.0 \\
58 / 16\end{array}$ \\
\hline 2 & $\begin{array}{l}\text { Net Transfer to recipient } \\
\text { Obs (On/off support) }\end{array}$ & $\begin{array}{c}+6.8 \\
132 / 38\end{array}$ & $\begin{array}{c}-4.8 \\
153 / 28\end{array}$ & $\begin{array}{l}-10.9 * * \\
259 / 23\end{array}$ & $\begin{array}{c}+4.2 \\
68 / 16\end{array}$ & $\begin{array}{l}-11.6 * * \\
79 / 14\end{array}$ & $\begin{array}{c}+9.6 \\
64 / 22\end{array}$ & $\begin{array}{c}+2.4 \\
74 / 14\end{array}$ \\
\hline
\end{tabular}

Stars indicate significance level of ATE using bootstrap standard errors, ${ }^{* * *} \mathrm{p}<0.01,{ }^{* *} \mathrm{p}<0.05,{ }^{*} \mathrm{p}<0.1$, exact matching on shock distribution, saving possibility / available insurance type and network strength. 
The descriptive and matching results clearly show a negative effect of the secret saving device on solidarity (Hypothesis III). Hence, the option to hide resources is used by individuals to reduce the social norm of providing transfers.

We also find a negative effect of insurance availability (Hypothesis I), but interestingly only if there is no secret saving. I.e. the negative effect is only found when shocks to co-players are fully observable. This is especially true for insurance option C. On the other hand, when the option of secret savings exists, and shocks are thus no longer fully observable, solidarity transfers are reduced greatly. But now the additional availability of insurance has no further detrimental effect on solidarity: The point estimates are insignificant and are even sometimes positive. ${ }^{34}$

We also find some evidence for a persistent effect of insurance availability. The availability of option $\mathrm{C}$ in round 1 reduces solidarity in round 2, i.e. after insurance has been removed (Hypothesis II). However, effects are not so clear for insurance type B.

As regards the results for our Hypothesis I, it is worthwhile to keep in mind that we do not yet know whether the effect is due to a crowding-out of motive or simply because insurance reduces inequality and thus lowers the need to redistribute. One cannot answer this question by the descriptive comparisons alone, as insurance on average implies a reduction in inequality. In the following analysis we will therefore try to separate these two channels.

\section{- Regression specification -}

So far, we have examined unconditional effects of insurance availability only, controlling for small sample imbalances in shocks via matching. For learning more about the possible explanatory channels, we need parametric regression models, given our small samples sizes. Using a regression model and controlling for differences in play money, we can disentangle the effects if insurance via reduced inequality and the additional crowding-out effects of insurance.

Via control variables, we can also control for small-sample imbalances in shock distributions, implied by the dice rolling, and for small-sample imbalances in individual characteristics. Furthermore we can also eliminate some statistical noise to reduce the large unexplained variation in transfers by including important background characteristics.

\footnotetext{
${ }^{34}$ Remember that the (insignificant) positive difference between treatment blocks $\mathrm{AB}$ and $\mathrm{A}$ in the first round might be explained by the lower amount that can be secretly saved with insurance $\mathrm{B}$.
} 
For specifying the regression model, we need to take into account that individual transfers are left-censored at zero because negative transfers are not allowed. ${ }^{35}$ We use Tobit regressions where the latent willingness to give is permitted to be proportional ${ }^{36}$ to the observable pre-transfer difference between giver and recipient. (I.e. the amount secretly saved by the recipient is not observable to the giver.) For visual comparison, Figure 4 shows a nonparametric fit of the relationship and the parametric Tobit regression fit. The comparison reveals that the Tobit regression fits the main relationship very well, with only a slight divergence at the both extremes.

Figure 4: Comparison between Tobit and nonparametric fit

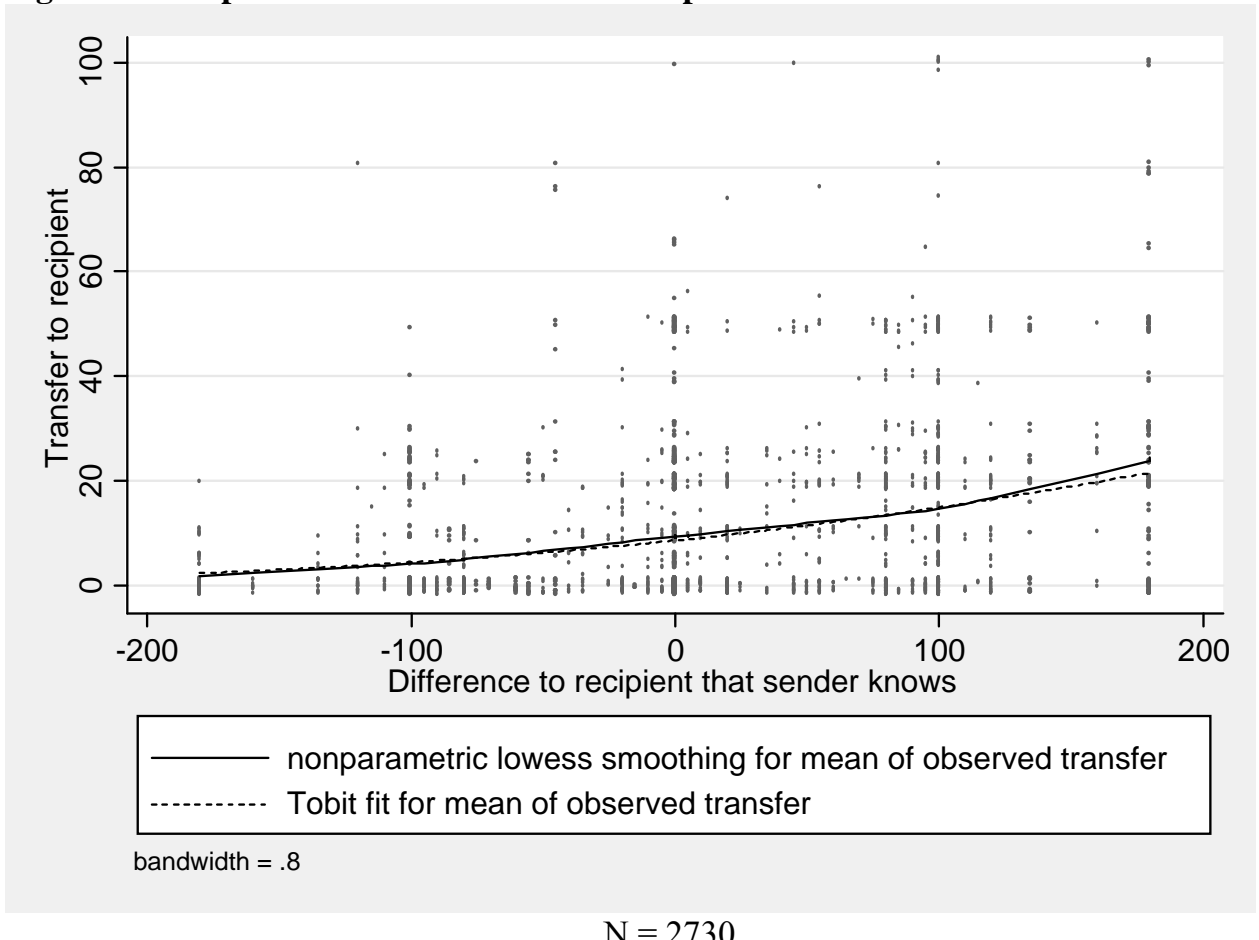

The following analysis consists of two parts. First, we examine the willingness to give, i.e. the willingness of one individual to transfer money based on private information and the observable information about the partners. We examine the transfer from individual $i$ to individual $\mathrm{j}$ in order to understand the willingness-to-give and its heterogeneity. Second,

\footnotetext{
${ }^{35}$ In principle, transfers are also right-censored as participants cannot transfer more than their money at hand. In reality, however, left-censoring is by far the most relevant problem. $57 \%$ of all transfers are zero, while only $2.5 \%$ percent of transfers are restricted by the money at hand. A two sample proportion test cannot reject equivalence of this proportion in the subsamples with and without secret saving $(3.0 \% \mathrm{vs} .2 .2 \%$, p-value $=0.17)$. ${ }^{36}$ We also tried non-linear terms, but these were insignificant.
} 
based on the estimates of the willingness to give we simulate the distributions of earnings, inequality and poverty under different scenarios. ${ }^{37}$

We consider the following regression model of individual transfers:

$$
T_{i j}^{*}=\left(\begin{array}{c}
Y_{i}-\left(Y_{j}-S_{j}\right) \\
Y_{i} \\
S_{i} \\
S_{j} \\
X_{i} \\
X_{j}
\end{array}\right)^{T} \beta+\varepsilon_{c, r}+\varepsilon_{i}
$$

where $T_{i j}^{*}$ is the latent transfer from $\mathrm{i}$ to $\mathrm{j}$

and

$$
\begin{array}{llll}
T_{i j}=T_{i j}^{*} & \text { if } & T_{i j}^{*} \geq 0 \\
T_{i j}=0 & \text { if } & T_{i j}^{*}<0
\end{array}
$$

Latent transfers are influenced by the difference in incomes between sender and recipient that the sender observes $\left(Y_{i}-Y_{j}\right)$. In case there is secret saving the sender can obviously only observe the recipient's income without savings $\left(Y_{i}-\left(Y_{j}-S_{j}\right)\right)$. We also allow an effect of own income after the lottery $\left(Y_{i}\right)$. Additionally including the saving amount $\left(S_{i}\right)$ is a direct test whether solidarity transfers are voluntary or obliged. If giving was truly intrinsically motivated, secret saving should not have any effect, because it changes the information available to the recipient - not to the sender. On the other hand, if transfers are driven by perceived group pressure, only the observable difference $\left(\left(Y_{i}-S_{i}\right)-\left(Y_{j}-S_{j}\right)\right)$ should matter.

On the other hand, including saving amounts of the recipient $\left(S_{j}\right)$ is a test whether savings of the recipient are really unobserved. ( $S_{j}$ is unobservable to $i$ by design, but during the communication stage person $j$ could have revealed some signals about his true shock or his secret savings $S_{j}$.)

In that case the observable difference should be all that matters and the coefficient should be zero otherwise. Individual covariates of sender/recipient $\left(X_{i}, X_{j}\right)$, community-round fixed

\footnotetext{
${ }^{37}$ For our analysis, we examine transfers from $\mathrm{i}$ to $\mathrm{j}$ instead of net transfers. Net transfers are a result of the decision of two individuals and thus the difference of two censored variables. Even if the underlying latent willingness is linear in regressors, the difference of the observed transfers will not be linear. (An exception is the case when all regressors for $T_{i j}$ are the negative of the regressors for $T_{j i}$. Linearity of the expected value could then be shown. This would for example be the case when the difference between sender and recipient was the only relevant explanatory, but we allow for many more influential factors.) The fact that net transfers are a function of the decisions by two people, who also have the option to transfer to a third person, implies that their error terms in their transfer decisions will be dependent, which would complicate the empirical analysis.
} 
effects $\left(\varepsilon_{c, r}\right)$ and an individual error term $\left(\varepsilon_{i}\right)$ are also allowed to affect $T_{i j}^{*}$. Note that all level effects that do not vary within a village-round cell are included in the fixed effect $\varepsilon_{c, r}$. This includes treatment, round and community effects. However, these level effects are common to both the sender and the receiver and are thus not of immediate interest.

Of particular interest to us will be the coefficient $\beta_{1}$, which indicates by how much voluntary transfers increase if income differences increase. If there is more solidarity of the better-off with the worse-off, transfers will be more sensitive to inequality. We are particularly interested in how solidarity changes with the availability of formal insurance. To this end we will incorporate interaction terms between $Y_{i}-\left(Y_{j}-S_{j}\right)$ and different treatment blocks and rounds.

\section{- Regression results -}

Table 8 shows the results of the Tobit regressions. Specification (1) is the base specification, regression (2) includes village-round controls and specification (3) adds individual covariates of sender and recipient. The latent willingness to give rises by an additional 0.13 pesos with $Y_{i}-\left(Y_{j}-S_{j}\right)$. The coefficient on $S_{i}$ is -0.11 , i.e. nearly of the same magnitude (the difference is statistically insignificant). Hence, if money is secretly saved by the sender, this almost oneto-one reduces the inclination to give - indicating that most of the solidarity is in fact not intrinsically motivated. Savings of the recipient on the other hand do not matter much. Remember that $S_{j}$ is unobserved to $i$, but some signals about intended behavior could have been given during the communication stage. The small coefficient on $S_{j}$ and the weaker significance level (as compared to the main effect) suggest that savings are largely unobserved, because the sender reacts more to the observable difference rather than to the real difference. It thus seems that mainly observable differences drive redistribution and money is saved in order to avoid peer pressure. 


\begin{tabular}{|c|c|c|c|c|c|c|}
\hline & (1) & $\begin{array}{c}\text { (2) } \\
\text { observatic }\end{array}$ & (3) & $\begin{array}{c}\text { (4) } \\
\text { All } \\
\text { observations }\end{array}$ & $\begin{array}{c}\text { (5) } \\
\text { Only } \\
\text { observations } \\
\text { without secret } \\
\text { saving option }\end{array}$ & $\begin{array}{c}\text { (6) } \\
\text { Only } \\
\text { observations } \\
\text { with secret } \\
\text { saving option } \\
\end{array}$ \\
\hline$Y_{i}$ & 0.047 & $0.048^{*}$ & $0.046^{*}$ & $0.048^{*}$ & 0.036 & $0.083^{*}$ \\
\hline Observable Difference $\left(Y_{i}-\left(Y_{j}-S_{j}\right)\right)$ & $0.13^{\star \star *}$ & $0.13^{\star \star *}$ & $0.13^{\star \star *}$ & $0.12^{\star \star *}$ & $0.15^{\star \star *}$ & 0.082 \\
\hline Saving sender $\left(S_{i}\right)$ & $-0.11^{\star * *}$ & $-0.13^{\star * *}$ & $-0.14 * \star$ & $-0.14^{\star \star \star}$ & & $-0.15^{\star \star *}$ \\
\hline Saving recipient $\left(S_{j}\right)$ & -0.018 & $-0.033^{*}$ & $-0.034^{*}$ & $-0.034^{*}$ & & $-0.026^{*}$ \\
\hline$\left(Y_{i}-\left(Y_{j}-S_{j}\right)\right) \times$ Treat B & & & & -0.020 & $-0.095^{\star *}$ & $0.052^{*}$ \\
\hline$\left(Y_{i}-\left(Y_{j}-S_{j}\right)\right) \times$ Treat $\mathrm{C}$ & & & & -0.021 & $-0.089^{\star *}$ & 0.033 \\
\hline$\left(Y_{i}-\left(Y_{j}-S_{j}\right)\right) \times$ Treat B $\times$ Treat C & & & & 0.060 & $0.15^{*}$ & -0.021 \\
\hline$\left(Y_{i}-\left(Y_{j}-S_{j}\right)\right) \times$ PseudoTreat B & & & & 0.025 & -0.032 & $0.098^{\text {«* }}$ \\
\hline$\left(Y_{i}-\left(Y_{j}-S_{j}\right)\right) \times$ PseudoTreat C & & & & -0.029 & $-0.11^{\star *}$ & 0.035 \\
\hline$\left(Y_{i}-\left(Y_{j}-S_{j}\right)\right) \times$ Round & & & & 0.015 & $0.030^{\star k}$ & -0.0058 \\
\hline$\left(Y_{i}-\left(Y_{j}-S_{j}\right)\right) \times$ Weaker Network & & & & -0.031 & -0.0092 & -0.011 \\
\hline Village-round controls & NO & YES & YES & YES & YES & YES \\
\hline Individual controls & NO & NO & YES & YES & YES & YES \\
\hline Observations & 2730 & 2730 & 2730 & 2730 & 1664 & 1066 \\
\hline
\end{tabular}

Standard errors in parentheses, clustered at the village level. ${ }^{* * *} \mathrm{p}<0.01,{ }^{* *} \mathrm{p}<0.05,{ }^{*} \mathrm{p}<0.1$

The coefficient for the control variables are shown in the Appendix (table A2).

Treat $\mathrm{B}$ is a dummy variable that indicates availability of insurance type B, It is 1 for villages in treatment block $\mathrm{AB}$ in round 1 as well as round 3 , and in block $\mathrm{A}$ in round 3. PseudoTreat $\mathrm{B}$ is a dummy variable that is 1 for villages in treatment block $\mathrm{AB}$ in round 2, i.e. where insurance had been available in the previous round but not in the current round.

Treat $\mathrm{C}$ and PseudoTreat $\mathrm{C}$ are defined analogously for villages in treatment block AC.

The interaction term (Treat B x Treat $\mathrm{C}$ ) is a dummy variable that is 1 for villages in treatment block $\mathrm{A}$ in round 3 only, where both insurance types where offered at the same time.

The regressor Round takes the values 1,2 and 3 .

The regressor Weaker network is a dummy variable that is 1 if the groups of three players had been formed at random.

The individual control variables are: Regular income, Skip meals last month, Debt larger than 1000 Pesos, Male, Household head, Male interacted with Household head, Married, High school, College, Age, Age squared. Both the sender's and the recipient's characteristics are included.

Village-round controls are: Treat B, Treat C, Treat B x Treat C, PseudoTreat B, PseudoTreat C, Round, Weaker Network, Income Class, partially urban, people are selfish

Columns (1) to (3) do not include any interaction terms between the observable pre-transfer difference $Y_{i}-\left(Y_{j}-S_{j}\right)$, and differ only in the set of control variables. Column (4) adds various interaction terms. Columns (5) and (6) use the same specification as in (4) but split the sample according to the availability of the secret saving device. The interaction term 
$\left(Y_{i}-\left(Y_{j}-S_{j}\right)\right) \times$ Treat $B$ reflects the different behavior in willingness to give once the insurance type B is available compared to the baseline scenario where no insurance exists. Similarly for the interaction term with Treat $C$, which reflects the availability of insurance type C. Remember that insurance $B$ is available in treatment block $A B$ in rounds 1 and 3 and in block $A$ in round 3. Insurance $C$ is available in treatment block $A C$ in rounds 1 and 3, and in block $\mathrm{A}$ in round 3. In treatment block $\mathrm{A}$ in round 3 , both insurance options are available at the same time. We capture this simultaneous availability of both options affects by the interaction term $\left(Y_{i}-\left(Y_{j}-S_{j}\right)\right)$ x Treat B x Treat $\mathrm{C}$, which is only one in block A round 3.

To examine possible effects of "making insurance once available and then withdrawing it", we introduce the dummies (PseudoTreat $B$ ) for treatment block $A B$ in round 2 and (PseudoTreat $C$ ) for treatment block $\mathrm{AC}$ in round 2. In both cases, insurance was available in round 1 but not in the current round. Please note that there is the comparison treatment block A where insurance was available in neither round. Hence, PseudoTreat is not confounded with the interaction term $\left(Y_{i}-\left(Y_{j}-S_{j}\right)\right)$ x Round, which measures general changes during the game. (The round numbers are 1, 2 and 3.) Finally, Weaker Network is a dummy variable which takes the value one if the group was formed at random.

Column (4) of Table 8 shows the estimation results with these interaction terms. Column (5) shows the same regression when using only those observations where secret saving was not possible, whereas column (6) uses only those observations where secret saving was possible. In column (4) we find that differences in pre-transfer earnings and savings of sender/recipient are again highly significant and similar in size to before. However, all interaction terms are insignificant. The interaction terms become significant though, once we account for the possibility of secret savings, columns (5) and (6).

Without the secret saving possibility (column 5), the availability of any insurance reduces the willingness-to-give by more than half. Also, the significantly negative interaction term with (PseudoTreat $C$ ) suggests a persistence of the effect even if insurance is removed in the second round. (The estimate for PseudoTreat $B$ is smaller and too imprecisely estimated to permit a clear interpretation.) We also find that participants become more sensitive to differences in $Y$ in later rounds, as indicated by the positive coefficient for $\left(Y_{i}-\left(Y_{j}-S_{j}\right)\right) x$ Round, when players become more accustomed with the game or their co-players. ${ }^{38}$

\footnotetext{
38 Remember that no feedback (on transfers or whatsoever) was given during the game. Players could communicate with each other, which increases trust and personal feelings and, although it is cheap talk, allows signaling their honesty and willingness to transfer. (All such signals are non-verifiable later, however.)
} 
In column (6), in contrast, only observations are used where secret saving was possible. Nearly all coefficients are insignificant. Only two interaction terms are significant at the $5 \%$ and $10 \%$ level. Yet, note that the sample size for column (6) is smaller than for column (5) such that the estimates are expected to be less significant. However, they are also less robust when repeating the same checks as for non-saving village-rounds (see below). ${ }^{39}$ Hence, when secret saving is possible, the availability of insurance does not seem to affect behavior in a systematic way.

In the following Table 9 we augment the previous regressions with more control variables in order to examine the robustness of the previous findings and also to learn more about variables that affect the willingness-to-give. Adding these covariates also controls for any small-sample imbalances in these regressors between the treatment blocks. First, we add more interactions between $Y_{i}-\left(Y_{j}-S_{j}\right)$ and individual/village covariates. We include all covariates that were at least marginally significantly unbalanced between treatment blocks. The results of Table 8 are fully confirmed and most additional control variables are insignificant. Interestingly, household heads have a substantially larger inclination to give transfers proportional to $Y_{i}-\left(Y_{j}-S_{j}\right)$ in the no-saving subsample, perhaps because of their general responsibility to balance needs within the household.

In columns (3) to (5) we examine various subsets of those participants where secret saving was not possible. In column (3) we exclude individuals with a lower level of understanding according to our test questions or because of particularly 'irrational' transfers. ${ }^{40}$ In columns (4) and (5) we examine the effects separately for each round. Since the sample sizes were quite small for each round, we always combine two rounds, i.e. rounds one and two in column (4) and rounds two and three in column (5). The coefficients are mostly significant for the more general insurance type B. This is not the case for catastrophicinsurance type C. While effects always point in the expected direction, they are insignificant. However, this is likely because of the limited sample size in the robustness checks. (We assess (in appendix III) whether the insignificant effect is due to limited sample size only or whether there is a systematic difference between the effects of insurance type B and $\mathrm{C}$. We conclude that there are no systematic differences.) The effect for type $\mathrm{C}$ again appears to be persistent if availability is removed (PseudoTreat $C$ ). The related coefficients are significant

\footnotetext{
${ }^{39}$ In particular, effects disappear when adding more controls (see table 9 column 1) or when excluding those with lower understanding and irrational transfers (see footnote 40).

${ }^{40}$ We define irrational transfers as transfers from individuals that lost more than some other group member but still transferred more than $40 \%$ of their money and received less from the others than what they gave.
} 
at least at the $5 \%$ level across all specifications. We cannot identify persistence of the effect for insurance B.

Table 9: Tobit regressions explaining transfer - further analyses

\begin{tabular}{|c|c|c|c|c|c|}
\hline & \multirow{2}{*}{$\begin{array}{c}\text { (1) } \\
\text { Only } \\
\text { observations } \\
\text { with secret } \\
\text { saving option }\end{array}$} & \multirow{2}{*}{$\begin{array}{c}\text { (2) } \\
\text { Only } \\
\text { observations } \\
\text { without secret } \\
\text { saving option }\end{array}$} & \multicolumn{3}{|c|}{$\begin{array}{c}\text { (3) } \\
\text { Only observations without secret } \\
\text { saving option }\end{array}$} \\
\hline & & & $\begin{array}{c}\text { High } \\
\text { understand }\end{array}$ & $\begin{array}{c}\text { Round } \\
1+2\end{array}$ & $\begin{array}{c}\text { Round } \\
2+3\end{array}$ \\
\hline$Y_{i}$ & $0.082^{*}$ & 0.036 & 0.035 & 0.028 & $0.061^{* *}$ \\
\hline Observable Difference $\left(Y_{i}-\left(Y_{j}-S_{j}\right)\right)$ & 0.0028 & 0.13 & $0.19^{\star \star *}$ & $0.14^{\star *}$ & $0.22^{\star * *}$ \\
\hline Saving sender $\left(S_{i}\right)$ & $-0.15^{\star \star *}$ & & & & \\
\hline Saving recipient $\left(S_{j}\right)$ & $-0.027^{*}$ & & & & \\
\hline$\left(Y_{i}-\left(Y_{j}-S_{j}\right)\right) \times$ Treat B & 0.041 & $-0.091^{* *}$ & $-0.12^{* *}$ & $-0.10^{* *}$ & -0.066 \\
\hline$\left(Y_{i}-\left(Y_{j}-S_{j}\right)\right) \times$ Treat $\mathrm{C}$ & 0.031 & $-0.096^{\star \star \star}$ & -0.083 & -0.041 & -0.095 \\
\hline$\left(Y_{i}-\left(Y_{j}-S_{j}\right)\right) \times$ Treat B x Treat C & -0.014 & $0.15^{\star \star}$ & 0.12 & & 0.14 \\
\hline$\left(Y_{i}-\left(Y_{j}-S_{j}\right)\right) \times$ PseudoTreat B & 0.086 & -0.020 & -0.049 & -0.029 & -0.049 \\
\hline$\left(Y_{i}-\left(Y_{j}-S_{j}\right)\right) \times$ PseudoTreat C & 0.029 & $-0.11 * *$ & -0.12 ** & $-0.11 *$ & $-0.12^{* *}$ \\
\hline$\left(Y_{i}-\left(Y_{j}-S_{j}\right)\right) \times$ Round & -0.0029 & $0.027^{*}$ & 0.021 & 0.041 & \\
\hline$\left(Y_{i}-\left(Y_{j}-S_{j}\right)\right) \times$ Weaker Network & -0.016 & -0.0012 & 0.010 & -0.026 & -0.012 \\
\hline$\left(Y_{i}-\left(Y_{j}-S_{j}\right)\right) \times$ Income Class & 0.020 & 0.020 & & & \\
\hline$\left(Y_{i}-\left(Y_{j}-S_{j}\right)\right) \times$ Selfish & -0.0043 & -0.0055 & & & \\
\hline$\left(Y_{i}-\left(Y_{j}-S_{j}\right)\right) \times$ Higher Education & 0.062 & $-0.035^{*}$ & & & \\
\hline$\left(Y_{i}-\left(Y_{j}-S_{j}\right)\right) \times \mathrm{HH}$ Head & -0.0045 & $0.054^{*}$ & & & \\
\hline Village-round controls & YES & YES & YES & YES & YES \\
\hline Individual controls & YES & YES & YES & YES & YES \\
\hline Observations & 1066 & 1664 & 1234 & 890 & 1216 \\
\hline
\end{tabular}

We additionally ran regressions allowing different crowding-out for groups formed at random (Weaker Network), but results are too imprecise to draw conclusions. Moreover, we tested whether the magnitude of crowding out depends on the number of insured players and also do not find significant effects (results available upon request).

Overall, empirical results suggest that there is a negative effect of insurance on solidarity if there is no secret saving possibility. Under these circumstances, the effect of the 
catastrophic-insurance is persistent even if insurance is removed. Furthermore, additional robustness checks show that, for both options $\mathrm{B}$ and $\mathrm{C}$, the availability of insurance is associated with lower inclination to redistribute and that the catastrophic-only insurance effect is more persistent than the effect of the comprehensive insurance type B. The more persistent effect of type $\mathrm{C}$ could be due to higher 'acceptance' amongst participants, given that the price of this catastrophic-insurance is lower and take-up is higher. At first glance this might hint at the importance of the information effect in explaining the crowding-out of solidarity. If many people choose insurance it signals that commitment or trust in the existing solidarity transfer scheme is low and thus provokes a negative response with low transfers. ${ }^{41}$ The stronger information effect would also explain differential persistence across rounds, as participants update their information about the co-players for the rest of the game. However, regressions controlling for insurance take-up do not reveal higher crowding out with higher take-up. Also, adoption of the catastrophic-insurance type $\mathrm{C}$ is not much higher in the first round, but rather in the third round (see figure 2). It should be the first round, however, that leads to persistence effects in the second round. Hence, the framing effect appears to be the main explanation for crowding-out, i.e. insurance availability signals that everybody is responsible for his/her own security. We discuss these results in some detail in section V.

\footnotetext{
${ }^{41}$ Similarly, if many people cheat with their tax declaration (and this is known to the rest of the population) it might give taxpayers a signal that compliance with the law is rather low which activates a reciprocal bandwagon effect ('people are honest conditional that others are honest') which weakens the norm of honesty further (Traxler 2010).
} 


\section{Simulation of poverty with insurance and/or secret saving possibility}

In the regressions we found substantial saving and insurance effects. Yet, the real importance of these effects is difficult to infer from the size of the coefficients alone. Transfers as well as the insurance options both lead to a reduction in the variance of the outcomes but their combined results cannot be obtained from the previous tables. ${ }^{42}$ In principle we could simply compare outcome distributions in different treatment combinations to obtain a meaningful comparison. In our small sample, however, such comparisons can be blurred by differences in dice results and other covariates. In addition, we are also interested in counterfactual simulations. We therefore use our previous regression results to simulate the counterfactual situation where everybody receives access to insurance treatment $(\mathrm{A} / \mathrm{AB} / \mathrm{AC})$ in all rounds. This is done separately for the regression results with and without secret savings, but always using the whole sample as a basis for the counterfactual. To effectively illustrate average treatment effects for a large population, we expand the datasets by the factor $100 .{ }^{43} \mathrm{We}$ draw shocks according to the theoretical probabilities, assign insurance take-up and secret saving (if applicable) according to the observed probabilities, and also draw error terms for the transfer decision from the estimated (normal) distribution. Given shocks, insurance uptake and secret saving decisions we can then predict transfers using our regression results (including random error terms). As a result we have a complete distribution of payoffs after insurance and informal solidarity for different counterfactual situations. ${ }^{44}$

In Table 10 we summarize our simulation results of the income distributions by examining the density mass below various "poverty lines". By examining the results for different poverty lines, we can see how robust our main findings are. The poverty line of 50 $\mathrm{PhP}$ corresponds to $25 \%$ of the initial endowment. Table 10 also shows the results for 40 and $60 \mathrm{PhP}$. For a poverty line of $50 \mathrm{PhP}$ the simulation results show that, when secret savings are not available, offering insurance type B changes the poverty rate from 6.6 to $7.8 \%$. Yet, when secret saving is possible, the poverty rates are 12.6 versus $8.2 \%$ with and without availability of insurance B. The respective numbers for insurance type $\mathrm{C}$ are $6.8 \%$ without savings, and $8.3 \%$ with secret savings. In the case of no secret savings we thus do not observe any positive effect of insurance compared with the case of no insurance provision. Vulnerability remains the same and taking into account administrative costs, access to insurance leads to lower

\footnotetext{
${ }^{42}$ Also note that these coefficients (effects on the latent willingness to give) are different from marginal effects (effects on the observed transfers). We abstain from calculating marginal effects, as they do not facilitate interpreting the results too much, contrary to the simulation results presented in this section.

${ }^{43}$ With such a large population variations of the outcome distribution by chance are very small. Repeating the simulation procedure 201 times leaves us with standard deviations of always less than 0.00115 using different points of the cumulative distribution function.

${ }^{44}$ Details on the simulation procedure can be found in Appendix II.
} 
welfare, e.g. for insurance type B in particular. The main conclusions hold also for the other poverty lines in Table 10. (We also examined other poverty lines, but do not show the results here as they did not provide additional insights. ${ }^{45}$ Table 10 also shows significance levels for testing whether the numbers in columns (2) and (3) are statistically different from those of column (1). Analogously, statistically differences between columns (5) and (6) to (1) are tested. Significance levels are obtained by bootstrapping the combined estimation and simulation process. We observe significant improvements when secret saving is possible, but no statistically significant differences when savings are not available.

Table 10: Poverty rates for different poverty lines under each saving/insurance regime (simulation)

\begin{tabular}{|c|c|c|c|c|c|c|}
\hline \multirow[b]{3}{*}{$\begin{array}{l}\text { Poverty } \\
\text { line at: }\end{array}$} & \multicolumn{3}{|c|}{ Without secret saving option } & \multicolumn{3}{|c|}{ With secret saving option } \\
\hline & (1) & (2) & (3) & (4) & (5) & (6) \\
\hline & $\begin{array}{c}\text { No } \\
\text { insurance } \\
\text { offered } \\
\end{array}$ & $\begin{array}{c}\text { Insurance } \\
\text { type B } \\
\text { offered } \\
\end{array}$ & $\begin{array}{c}\text { Insurance } \\
\text { type C } \\
\text { offered } \\
\end{array}$ & $\begin{array}{c}\text { No } \\
\text { insurance } \\
\text { offered } \\
\end{array}$ & $\begin{array}{c}\text { Insurance } \\
\text { type B } \\
\text { offered } \\
\end{array}$ & $\begin{array}{c}\text { Insurance } \\
\text { type C } \\
\text { offered }\end{array}$ \\
\hline $40 \mathrm{PhP}$ & $4.6 \%$ & $6.0 \%$ & $4.8 \%$ & $9.6 \%$ & $5.6 \% * *$ & $5.6 \% * *$ \\
\hline $50 \mathrm{PhP}$ & $6.6 \%$ & $7.8 \%$ & $6.8 \%$ & $12.6 \%$ & $8.2 \% * *$ & $8.3 \% * *$ \\
\hline $60 \mathrm{PhP}$ & $9.4 \%$ & $10.0 \%$ & $9.0 \%$ & $16.0 \%$ & $11.6 \% * *$ & $11.8 \% * *$ \\
\hline
\end{tabular}

Whereas Table 10 was based on simulation results, for comparison we also show in Table 11 the observed "poverty rates" in our data. Since our simulations in Table 10 are based on regressions estimated from these data, both tables should show similar patterns. In principle, Tables 10 and 11 should show the same numbers for large N. Due to the small sample size, the numbers in Table 11 partly also reflect differences in the shock and covariate distributions across the treatments, therefore the patterns are more noisy than in Table 10. The general tendency of the simulation can be confirmed. Poverty rates are generally lower in the secret saving case and the situation is less clear without secret savings. (Table 11 shows the numbers for round 1 , because circumstances are most comparable to the simulated situation above.)

The phenomenon that without secret saving the unlucky are on average not better protected if there is insurance of one type also very directly shows up in another statistic: The poverty rate in the data (at 50 PHP) in the data among those who had a catastrophic shock is

\footnotetext{
${ }^{45}$ The complete distributions of payoffs after transfers under different insurance schemes can be found in Figures $\mathrm{A} 1 \mathrm{a}$ and A1b in Appendix I.
} 
not significantly lower when insurance type $\mathrm{B}$ or $\mathrm{C}$ is available $(30 \% \mathrm{vs} .31 \%)$ compared to in the absence of any insurance (38\%). The result changes if secret saving is possible. Poverty rate amongst the very unlucky is now much lower with access to insurance type B or C (23\% vs. $33 \%$ ) than without any insurance (64\%). This later result is significant at the $1 \%$ level for type $\mathrm{B}$ and at the $10 \%$ level for type $\mathrm{C}$, using a two-group test of proportion. ${ }^{46}$

Table 11: Observed poverty rates for different poverty lines under different regimes in round 1

\begin{tabular}{l|ccc|ccc}
\hline \hline & \multicolumn{2}{|c|}{ Without secret saving option } & \multicolumn{3}{c}{ With secret saving option } \\
$\begin{array}{l}\text { Poverty } \\
\text { line at: }\end{array}$ & $\begin{array}{c}\text { No } \\
\text { insurance } \\
\text { offered }\end{array}$ & $\begin{array}{c}\text { Insurance } \\
\text { type B } \\
\text { offered }\end{array}$ & $\begin{array}{c}\text { Insurance } \\
\text { type C } \\
\text { offered }\end{array}$ & $\begin{array}{c}\text { No } \\
\text { insurance } \\
\text { offered }\end{array}$ & $\begin{array}{c}\text { Insurance } \\
\text { type B } \\
\text { offered }\end{array}$ & $\begin{array}{c}\text { Insurance } \\
\text { type C } \\
\text { offered }\end{array}$ \\
\hline $40 \mathrm{PhP}$ & $1.6 \%$ & $7.3 \%$ & $0.0 \%$ & $9.2 \%$ & $3.6 \%$ & $0.0 \%$ \\
\hline $50 \mathrm{PhP}$ & $4.7 \%$ & $7.3 \%$ & $2.6 \%$ & $12.3 \%$ & $3.6 \%$ & $4.7 \%$ \\
\hline $60 \mathrm{PhP}$ & $9.4 \%$ & $8.5 \%$ & $6.4 \%$ & $14.6 \%$ & $4.8 \%$ & $9.4 \%$ \\
\hline \hline
\end{tabular}

To summarize the findings from Table 10: As long as there is no secret saving option, the informal insurance system seems to work rather well. The poverty rates with the formal insurance option $\mathrm{C}$ are the same as without any formal insurance option; see columns (1) and (3). An expensive insurance product (i.e. option B) may only do harm and increase poverty a little in column (2).

The introduction of secret savings (i.e. limited observability of shocks) has large effects, though. It reduces the willingness-to-transfer dramatically and the informal insurance system "breaks down". As long as no formal insurance options are available poverty rates almost double (compare column (4) to (1)). Now, providing the option of formal insurance helps to reduce inequality and poverty almost to levels without secret saving (compare column (5) and (6) to column (4) and (1), respectively).

\footnotetext{
${ }^{46}$ Note that these comparisons are not necessarily balanced as the observations come from different rounds (see the treatment plan in table 2). A balanced comparison is possible when restricting the sample to observations in the first round. Even though we have a small number of catastrophic shocks ( $N=52$ compared to $N=169$ before) when looking at round one only we still find the same qualitative result. The poverty rate is lower with insurance $\mathrm{B} / \mathrm{C}$ available if there is secret saving and the differences are significant at the $1 \% / 5 \%$ level. Without secret saving the difference is insignificant.
} 


\section{- Counterfactual simulations -}

The inefficiency of insurance in the case without secret savings has at least two aspects worth exploring. First, crowding out reduces the potential of the informal solidarity transfer mechanism. But how substantial is this effect? How efficient would the insurance products be without crowding out? Second, the effectiveness of insurance is limited by incomplete uptake. If insurance availability crowds out solidarity transfers and some individuals remain uninsured those are even more vulnerable. How would mandatory insurance work in such a context? Would this decrease vulnerability? In the following we thus provide hypothetical simulation results with mandatory insurance (100\% uptake) and with absence of crowding out. (As before, the simulations are based on the regression specifications of Table 9.)

Table 12 shows poverty risk under different insurance/saving schemes if there was no crowding out effect of insurance. No crowding out effect means assuming the same sensitivity of transfers to payoff differences with and without insurance. Looking at the left part of the table (no secret saving), we can now observe a decrease of poverty rates at all poverty lines when insurance becomes available. ${ }^{47}$ This is contrary to the effects in table 10 because crowding out is disabled in Table 12.

On the other hand, the numbers on the right side of table 12 (with secret saving) are more similar to (the right hand side of) table 10. Since we did not find significant crowding out when hiding money was possible, it is not surprising that setting the crowding-out effect to zero does not make a large difference. (Note that the poverty rates in the right hand side of Table 12 are somewhat larger than in Table 10, because the estimated coefficients in column (1) of Table 9 were, although not being statistically significant, nevertheless positive. ${ }^{48}$ The effects on the full distributions are shown in the appendix (figures A2a and A2b).

Table 13 illustrates the effect of introducing a mandatory insurance scheme. We assume the same degrees of crowding out as in table $10 .^{49}$ The effects at all poverty lines and

\footnotetext{
${ }^{47}$ The effect at all poverty lines can be seen from Figures A2a and A2b in the Appendix (showing complete distributions under different schemes).

${ }^{48}$ Also note that the simulated numbers of Table 12 are less noisy than those of Table 10 because fewer coefficients were estimated in each simulation replication since the coefficients measuring crowding out were fixed at zero.

${ }^{49}$ The only difference to Table 10 is that we set insurance uptake to one for the whole sample. Everything else remains unchanged in the simulation procedure. This simulation is therefore speculative because the introduction of mandatory insurance could also change the magnitude of the crowding out. With universal insurance the feeling of responsibility for other individuals could for example decrease further. On the other hand, with universal insurance income differences can no longer be attributed to a lack of precaution, but are purely
} 
under both saving schemes are very large. In fact, comparing Table 13 to Table 12, we find that full uptake with crowding out has in all cases a much larger effect than voluntary insurance without crowding out. The effects on the full distributions are shown in the appendix (figures A3a and A3b).

Table 12: Poverty rates for different poverty lines- without crowding out effect (simulation)

\begin{tabular}{l|ccc|ccc}
\hline \hline & \multicolumn{2}{|c|}{ Without secret saving option } & \multicolumn{3}{c}{ With secret saving option } \\
$\begin{array}{l}\text { Poverty } \\
\text { line at: }\end{array}$ & $\begin{array}{c}\text { No } \\
\text { insurance } \\
\text { offered }\end{array}$ & $\begin{array}{c}\text { Insurance } \\
\text { type B } \\
\text { offered }\end{array}$ & $\begin{array}{c}\text { Insurance } \\
\text { type C } \\
\text { offered }\end{array}$ & $\begin{array}{c}\text { No } \\
\text { insurance } \\
\text { offered }\end{array}$ & $\begin{array}{c}\text { Insurance } \\
\text { type B } \\
\text { offered }\end{array}$ & $\begin{array}{c}\text { Insurance } \\
\text { type C } \\
\text { offered }\end{array}$ \\
\hline $40 \mathrm{PhP}$ & $4.6 \%$ & $4.0 \% *$ & $3.0 \% * * *$ & $9.6 \%$ & $6.6 \% * * *$ & $6.3 \% * * *$ \\
\hline $50 \mathrm{PhP}$ & $6.6 \%$ & $5.6 \% * *$ & $4.8 \% * * *$ & $12.6 \%$ & $9.4 \% * * *$ & $9.1 \% * * *$ \\
\hline $60 \mathrm{PhP}$ & $9.4 \%$ & $7.9 \% * * *$ & $6.9 \% * * *$ & $16.0 \%$ & $12.8 \% * * *$ & $12.7 \% * * *$ \\
\hline \hline
\end{tabular}

Note: Stars indicate significance of the difference to the 'no insurance' state, based on bootstrapped simulation with 200 repetitions. Crowding out is disabled by setting the coefficients for $\left(Y_{i}-\left(Y_{j}-S_{j}\right)\right) \times$ Treat B and

$$
\left(Y_{i}-\left(Y_{j}-S_{j}\right)\right) \times \text { Treat } \mathrm{C} \text { to zero. }
$$

Table 13: Poverty rates for different poverty lines- mandatory insurance (simulation)

\begin{tabular}{l|ccc|ccc}
\hline \hline & \multicolumn{2}{|c|}{ Without secret saving option } & \multicolumn{3}{c}{ With secret saving option } \\
$\begin{array}{l}\text { Poverty } \\
\text { line at: }\end{array}$ & $\begin{array}{c}\text { No } \\
\text { insurance } \\
\text { offered }\end{array}$ & $\begin{array}{c}\text { Insurance } \\
\text { type B } \\
\text { mandatory }\end{array}$ & $\begin{array}{c}\text { Insurance } \\
\text { type C } \\
\text { mandatory }\end{array}$ & $\begin{array}{c}\text { No } \\
\text { insurance } \\
\text { offered }\end{array}$ & $\begin{array}{c}\text { Insurance } \\
\text { type B } \\
\text { mandatory }\end{array}$ & $\begin{array}{c}\text { Insurance } \\
\text { type C } \\
\text { mandatory }\end{array}$ \\
\hline $40 \mathrm{PhP}$ & $4.6 \%$ & $1.0 \% * * *$ & $0.8 \% * * *$ & $9.6 \%$ & $2.2 \% * * *$ & $2.3 \% * * *$ \\
\hline $50 \mathrm{PhP}$ & $6.6 \%$ & $2.0 \% * * *$ & $2.2 \% * * *$ & $12.6 \%$ & $3.9 \% * * *$ & $4.9 \% * * *$ \\
\hline $60 \mathrm{PhP}$ & $9.4 \%$ & $3.9 \% * * *$ & $4.2 \% * * *$ & $16.0 \%$ & $6.9 \% * * *$ & $8.3 \% * * *$ \\
\hline \hline
\end{tabular}

Note: Complete insurance take-up and crowding out as under insurance choice is assumed.

Stars indicate significance of the difference to the 'no insurance' state,

based on bootstrapped simulation with 200 repetitions

random. This might even foster the feeling of responsibility and increase the willingness to provide transfers to the poorer. Hence, the change in crowding out with mandatory insurance could go either way. 


\section{Discussion and Conclusions}

Informal risk-sharing is frequent in many developing countries, but usually cannot offer full protection because of limited enforcement mechanisms. In addition, even if people would voluntarily want to help each other in case of shocks they may not be able to do so in the case of covariate shocks, e.g. if the entire network is affected by a disease or other catastrophe. This gives rise to demand the introduction of formal insurance products tailored to the needs of the poor. In this paper we present a novel behavioral experiment with rural and partially urban villagers on the Philippines. This experiment - simulating a risky environment with solidarity networks and the introduction of anonymous insurance - delivers the first experimental evidence on whether informal solidarity is reduced by formal insurance in developing countries.

Our data highlight that the availability of insurance reduces solidarity and that this negative effect might even persist if insurance is removed. The latter finding is also particularly relevant for policy guidance in indicating that a flawed introduction of formal insurance products, e.g. of too expensive or inappropriate insurance that is later withdrawn, can have negative long-term consequences. The empirical analyses revealed that the negative effect is not only due to lower inequality between those with insurance but that there is an additional crowding-out effect on solidarity. However, this is only the case if shocks of network members are observable.

Once the shocks are no longer fully observable, which we introduce via a secret savings options, the solidarity system breaks down substantially. So why do effects only exist when shocks are observable? One important observation at this point is that nearly all people (94\%) pretend a shock and secretly save if there is the possibility to do so. As a consequence solidarity transfers are reduced dramatically (see Table 4). A lot of the solidarity transfers thus are probably motivated by "internalized peer pressure" rather than altruism. ${ }^{50}$ With solidarity transfers being so low, observing further reductions is hard. Hence, introducing the option of formal insurance cannot lower the already low solidarity transfers so much more. This might very well explain why the insurance effect is found in the non-saving villages where there is still solidarity in place.

\footnotetext{
${ }^{50}$ Note that transfers are never revealed. Consequently, there should be no fear of punishment or explicit group pressure. The only thing that changes when secretly saving money is that the recipient in need does not know about the potential resources available to the sender. Hence, the expectation levels regarding transfers are decreased, something that might be internalized by the sender who uses the secret saving device.
} 
Regarding the more persistent negative effect of a catastrophic-only product (C) versus a more comprehensive scheme (B) we observe considerably lower take-up of the more comprehensive scheme. Thus, participation might be too low for type B to induce a 'common sense' or 'social norm' that the market mechanism should apply. The so-called 'framing effect' means that once good formal insurance products become available it then becomes socially accepted that everyone is responsible on her own for insurance. Once such a social norm has evolved the moral obligation to provide voluntary transfers to people who willingly have not bought insurance dissipates quickly. Before such changes can be expected, a "good" insurance product must exist for most people. Judging from the take-up rates, insurance option C appears to be closer to a "good" product than option B. Some speculation allowed this could have to do with the relatively high price of option B. While everybody with reasonably high risk aversion can be expected to purchase the catastrophic-only insurance $\mathrm{C}$ this is not the case for the more expensive version B, which would be appealing only to very risk-averse persons. A simple simulation using a constant relative risk aversion (CRRA) utility function $\left[u(c)=\left(c^{1-\rho}\right) /(1-\rho)\right]$, where risk-averse individuals have $\rho>0$, shows that, in the absence of any solidarity transfers, all people with a risk-aversion parameter $\rho>0.34$ would buy insurance $\mathrm{C}$ if available, whereas only people with $\rho>0.65$ would buy insurance B. When comparing take-up of insurance type B and C in round one and three (figure 2) we would consequently classify around $35-40 \%$ as highly risk averse $(\rho>0.65)$, 5$16 \%$ as moderately risk averse $(0.34<\rho<0.65)$ and $50-55 \%$ as less risk averse $(\rho<0.34)$. Interestingly, Holt and Laury (2002, p. 1649) in a high stake experiment estimate the following proportions: $39 \%$ with $\rho>0.68,23 \%$ with $0.41<\rho<0.68,38 \%$ with $\rho<0.41$. Even though the comparison of our participants with US students might be a little far-fetched, the proportions are similar. ${ }^{51}$ Thus it seems plausible that insurance type $\mathrm{C}$ is considered a more convincing alternative.

An alternative explanation of the observed behavior could be the information effect. Stronger crowding-out with higher take-up would also be in line with the fact that reciprocity is important for risk-sharing and that buying insurance signals low trust or commitment in the informal risk-sharing mechanism. This 'information effect' could apply if individuals observe the insurance-buying behavior of their co-players in previous rounds and then form expectations about the solidarity/selfishness-types of their co-players. This could then lead to

\footnotetext{
${ }^{51}$ Cardenas and Carpenter (2008) provide a comparison of CRRA parameter estimates in developing countries and state: "There is some variation in the results (Table 5) but it is not explained by development. ... Overall, there does not appear to be much support for the idea that poor people in developing countries are more risk averse than richer people in developed countries." (Cardenas and Carpenter 2008, p. 326)
} 
a negative bandwagon effect with low transfers. Yet, we do not find that the impact increases with the number of insured in the group which should be the case when the information effect would be the main driver. Also, take-up of the insurance types differs mainly in the third round, which cannot affect information in the second round. Thus, the information effect is unlikely to be the main or the only effect.

In sum, our experimental results suggest that the introduction of insurance in solidarity networks might have unintended consequences under some circumstances. Especially if the network is able to observe the cash flow of members and reciprocal solidarity works well, these effects have to be taken into account. Short- and long-run effects are in line with the general literature on crowding-out of pro-social behavior by market based mechanisms (Bowles 2008).

However, formal insurance might very well have considerable positive net effects, because informal solidarity is not always very effective. If network members can pretend shocks, secretly save and thereby retreat from their solidarity commitment, availability of formal insurance can be a considerable improvement for individuals. In our experiment almost all (94\%) people used the possibility to pretend shocks. This overwhelming use of pretending shocks may indicate that also in real life people use (or like to use) devices to pretend shocks, hide money or pretend to be illiquid. Innovations such as mobile banking may add to making wealth less observable: At least in rural areas, wealth and assets such as the number of cattle and livestock, the quality of crops, the size of landholdings, the size and quality of the house etc are easily observable to everyone, whereas savings on a mobile phone would be invisible.

The partial ineffectiveness of insurance supply to protect against poverty heavily hinges on incomplete take-up. Participants below the poverty line in insurance treatments are mostly non-buyers. ${ }^{52}$ Thus our experimental results suggest that in a world without saving products (as is still the case for a large majority of people in developing countries) it can be better to have no voluntary insurance at all or alternatively force everybody into a compulsory public insurance scheme. Otherwise, there might always be a considerable fraction without insurance, as our data shows for every round. Although solidarity transfers are reduced by the access to insurance this reduction is 'not sufficient' in order to voluntarily bring the uninsured individuals into voluntary insurance schemes. As pointed out by Buchanan (1975) helping somebody may undermine his or her incentive to care for him or herself (i.e. insure). As long

\footnotetext{
${ }^{52} 87 \%$ of participants below the poverty line in treatments with insurance access were non-adopters.
} 
as there are enough 'Samaritans' with altruistic motives that help people in need (even though there is the possibility to insure against risks) and the 'Samaritans' are unable to commit not to provide help to uninsured individuals who face a loss, there will be an undesired underinsurance (compared with compulsory insurance) together with a crowding-out effect (compared to the no-insurance case). We also conducted various hypothetical counterfactual simulation analyses. These simulations indicated that mandatory insurance could solve the coordination problem and lead to reductions in vulnerability (see table 13).

While the above made statement favoring compulsory insurance rests on some assumptions (e.g. stability of crowding out over time, persistently incomplete take-up and other issues regarding external validity of our experiment) our main conclusion is that financial products serve people most when they are offered as a bundle. Introducing insurance in contexts without (formal) banking, with good monitoring in the network and strong informal solidarity might well lead to unintended consequences and could even be harmful. However, the story is different when saving options are available or will be introduced. Even though it might seem that introducing (secret) saving plays a harmful role in our experiment there are many good reasons to introduce such financial products. Especially, people will be able to use their savings for intertemporal income smoothing (or for saving for lumpy investments such as buying refrigerators, bicycle etc), an aspect we completely excluded up to now. In such situations, the combination of both financial products can be effective. While saving acts as an intertemporal smoothing device, insurance can compensate its negative side effect on risk-sharing within the network. Together, access to insurance and saving can then decrease vulnerability. 


\section{References}

Ashraf, Nava, Dean Karlan, and Wesley Yin. 2006. "Tying Odysseus to the Mast: Evidence from a Commitment Savings Product in the Philippines." Quarterly Journal of Economics 121 (2): 635-672.

Attanasio, Orazio, and José-Víctor Ríos-Rull. 2000. "Consumption smoothing in island economies: Can public insurance reduce welfare?" European Economic Review 44 (7): $1225-1258$.

Banerjee, A.V., and Esther Duflo. 2007. "The economic lives of the poor.” Journal of Economic Perspectives 21 (1): 141-167.

Barr, Abigail, and Garance Genicot. 2008. "Risk sharing, commitment, and information: an experimental analysis." Journal of the European Economic Association 6 (6): 11511185.

Besley, Timothy. 1995. "Savings, credit and insurance." Handbook of development economics 3: 2123-2207.

Bowles, Samuel. 2008. "Policies designed for self-interested citizens may undermine "the moral sentiments': Evidence from economic experiments." Science 320 (5883): 16051609.

Brune, Lasse, Xavier Giné, Jessica Goldberg, and Dean Yang. 2011. "Commitments to Save: A Field Experiment in Rural Malawi." World Bank Policy Research Working Paper 5748.

Buchanan, James McGill. 1975. The Samaritan's Dilemma. In Altruism, morality and economic theory, ed. E. Phelps, 71-85. New York: Russell Sage Foundation.

Cardenas, Juan Camilo, and Jeffrey Carpenter. 2008. "Behavioural Development Economics: Lessons from Field Labs in the Developing World." Journal of Development Studies 44 (3): 311-338.

Chandrasekhar, Arun G., Cynthia Kinnan, and Horacio Larreguy. 2011. Informal Insurance, Social Ties, and Financial Development: Evidence from a Lab Experiment in the Field. http://www.aae.wisc.edu/mwiedc/papers/2011/Kinnan_Cynthia.pdf.

Churchill, Craig. 2006. Protecting the poor: A microinsurance compendium. Geneva: International Labour Office.

Coate, Stephen, and M. Ravallion. 1993. "Reciprocity without commitment: Characterization and performance of informal insurance arrangements." Journal of development Economics 40 (1): 1-24.

Cole, S.A., X. Giné, J. Tobacman, P.B. Topalova, R.M. Townsend, and J.I. Vickery. 2009. "Barriers to household risk management: evidence from India." Harvard Business School Working Paper 09-116. 
Comola, Margherita, and Marcel Fafchamps. 2010. "Are gifts and loans between households voluntary?" CSAE Working Paper 2010-20.

Dercon, Stefan, and Pramila Krishnan. 2003. "Risk sharing and public transfers." The Economic Journal 113 (486): C86-C94.

Dupas, P., and Jonathan Robinson. 2009. "Savings constraints and microenterprise development: Evidence from a field experiment in Kenya." NBER Working Paper 14693.

Fafchamps, Marcel. 2008. Risk sharing between households. In Handbook of Social Economics, ed. Jess Benhabib, Alberto Bisin, and Matthew O. Jackson, 1-42. Elsevier.

Fafchamps, Marcel, and Flore Gubert. 2007a. "Contingent Loan Repayment in the Philippines.” Economic Development and Cultural Change 55 (4): 633-667.

- 2007b. “The formation of risk sharing networks." Journal of Development Economics 83 (2): 326-350.

Fafchamps, Marcel, and Susan Lund. 2003. "Risk-sharing networks in rural Philippines." Journal of Development Economics 71 (2): 261-287.

Flory, Jeffrey A. 2011. Modern Institutions \& Pre-Modern Safety Nets: Indirect Effects of Formal Savings Expansion on the "Unbanked" and Ultra-Poor. http://www.aae.wisc.edu/mwiedc/papers/2011/Flory_Jeff.pdf.

Giné, Xavier, and Dean Yang. 2009. "Insurance, Credit, and Technology Adoption: Field Experimental Evidence from Malawi." Journal of Development Economics 89 (1): 1-11.

Grimm, Michael, Flore Gubert, Jann Lay, and Christophe J Nordman. 2011. Kinship-ties and entrepreneurship in Western Africa.

http://campus.iss.nl/ grimm/ForcedSolidarity_V2.pdf.

Harrison, G.W., and E.E. Rutström. 2008. "Risk aversion in the laboratory." Research in Experimental Economics 12: 41-196.

Hoff, K, and A Sen. 2006. The kin system as a poverty trap? In Poverty Traps, ed. Samuel Bowles, Steven N. Durlauf, and Karla Hoff, 95-115. Princeton, Woodstock, New York: Princeton University Press.

Holt, Charles A, and Susan K Laury. 2002. "Risk Aversion and Incentive Effects." American Economic Review 92 (5): 1644-1655.

Ito, Seiro, and H. Kono. 2010. "Why is the take-up of microinsurance so low? Evidence from a health insurance scheme in India." The Developing Economies 48 (1): 74-101.

Jowett, Matthew. 2003. "Do informal risk sharing networks crowd out public voluntary health insurance? Evidence from Vietnam.” Applied Economics 35 (10): 1153-1161.

Kahneman, Daniel, and Amos Tversky. 1979. "Prospect theory: An analysis of decision under risk." Econometrica 47 (2): 263-291. 
Morduch, Jonathan. 1999. "Between the state and the market: Can informal insurance patch the safety net?” The World Bank Research Observer 14 (2): 187-207.

Platteau, Jean-Philippe. 1997. "Mutual insurance as an elusive concept in traditional rural communities.” Journal of Development Studies 33 (6): 764-796.

. 2000. Institutions, Social Norms and Economic Development. Amsterdam: Routledge.

Roth, Jim, Michael J. McCord, and Dominic Liber. 2007. The landscape of microinsurance in the world's 100 poorest countries.

http://www.microinsurancecentre.org/resources/documents/doc_download/634-thelandscape-of-microinsurance-in-the-worlds-100-poorest-countries-in-english.html.

Selten, R, and A. Ockenfels. 1998. “An experimental solidarity game.” Journal of Economic Behavior \& Organization 34 (4): 517-539.

Townsend, R.M. 1994. "Risk and insurance in village India.” Econometrica 62 (3): 539-591.

Traxler, Christian. 2010. "Social norms and conditional cooperative taxpayers." European Journal of Political Economy 26 (1): 89-103.

Trhal, Nadja, and Ralf Radermacher. 2009. "Bad luck vs. self-inflicted neediness - An experimental investigation of gift giving in a solidarity game." Journal of Economic Psychology 30 (4): 517-526. 


\section{Appendix I: Tables}

Table A1: Descriptive statistics of villages

\begin{tabular}{|c|c|c|c|c|c|c|c|}
\hline & \multicolumn{4}{|l|}{$\begin{array}{l}\text { All } \\
(N=22)\end{array}$} & \multirow{2}{*}{$\begin{array}{l}A \\
(\mathrm{~N}=6) \\
\text { Mean }\end{array}$} & \multirow{2}{*}{$\begin{array}{l}\mathrm{AB} \\
(\mathrm{N}=8) \\
\text { Mean }\end{array}$} & \multirow{2}{*}{$\begin{array}{l}A C \\
(N=8) \\
\text { Mean }\end{array}$} \\
\hline & Mean & Std. & Min & Max & & & \\
\hline How many people live in this community? & 1264 & 653 & 350 & 3123 & 1445 & 1284 & 1109 \\
\hline $\begin{array}{l}\text { How many different religious groups } \\
\text { in this village }\end{array}$ & 2.45 & 1.26 & 1 & 5 & 2.67 & 2.5 & 2.25 \\
\hline Households with family members abroad & $9.2 \%$ & $9.0 \%$ & 0 & $34.5 \%$ & $7.5 \%$ & $13.0 \%$ & $6.7 \%$ \\
\hline $\begin{array}{l}\text { Conflicts between people } \\
\text { (0=none, } 1=a \text { little, } 2=a \text { lot) }\end{array}$ & 1.50 & 0.67 & 0 & 2 & 1.33 & 1.88 & 1.25 \\
\hline Number of village organizations & 7.23 & 1.66 & 4 & 11 & 7.83 & 6.88 & 7.13 \\
\hline $\begin{array}{l}\text { People are selfish } \\
\text { (0 [abs. agree] - } 10 \text { [abs. disagree]) }\end{array}$ & 6.36 & 2.97 & 0 & 10 & 5 & $7.88^{*}$ & 5.88 \\
\hline $\begin{array}{l}\text { Trust to lend/borrow } \\
\text { (0 [abs. agree] - } 10 \text { [abs. disagree]) }\end{array}$ & 5.27 & 3.18 & 0 & 10 & 5 & 5.38 & 5.38 \\
\hline $\begin{array}{l}\text { Always somebody willing to help } \\
\text { (0 abs. agree - } 10 \text { abs. disagree) }\end{array}$ & 7.95 & 2.36 & 0 & 10 & 8.17 & 8.13 & 7.63 \\
\hline $\begin{array}{l}\text { Income Class } \\
\text { (officially assigned: } 1 \text { richest }-5 \text { poorest) }\end{array}$ & 3.45 & 0.60 & 3 & 5 & 3 & $3.75^{\star \star}$ & $3.5^{\star \star}$ \\
\hline 1=partially urban / $0=$ rural & 0.68 & & 0 & 1 & 0.5 & 0.75 & 0.75 \\
\hline
\end{tabular}

Table A2: Regressions fromTable 8 with individual and village/round covariate coefficients

\begin{tabular}{|c|c|c|c|c|c|c|}
\hline & (1) & (2) & (3) & $\begin{array}{c}\text { (4) } \\
\text { All } \\
\text { observations }\end{array}$ & $\begin{array}{c}\text { (5) } \\
\text { Only observations } \\
\text { without secret } \\
\text { saving option } \\
\end{array}$ & $\begin{array}{c}\text { (6) } \\
\text { Only observations } \\
\text { with secret saving } \\
\text { option }\end{array}$ \\
\hline$Y_{i}$ & 0.047 & $0.048^{*}$ & $0.046^{*}$ & $0.048^{*}$ & 0.036 & $0.083^{*}$ \\
\hline Observable Difference $\left(Y_{i}-\left(Y_{j}-S_{j}\right)\right)$ & $0.13^{\star \star \star}$ & $0.13^{\star \star \star}$ & $0.13^{\star \star k}$ & $0.12^{\star \star *}$ & $0.15^{\star * *}$ & 0.082 \\
\hline Saving sender $\left(S_{i}\right)$ & $-0.11 * * \star$ & $-0.13^{\star \star \star}$ & $-0.14^{* * *}$ & $-0.14^{\star \star *}$ & & $-0.15^{\star \star \star}$ \\
\hline Saving recipient $\left(S_{j}\right)$ & -0.018 & $-0.033^{\star}$ & $-0.034^{*}$ & $-0.034^{*}$ & & $-0.026^{*}$ \\
\hline$\left(Y_{i}-\left(Y_{j}-S_{j}\right)\right) \times$ Treat B & & & & -0.020 & $-0.095^{\star *}$ & $0.052^{*}$ \\
\hline$\left(Y_{i}-\left(Y_{j}-S_{j}\right)\right) \times$ Treat $\mathrm{C}$ & & & & -0.021 & $-0.089 * *$ & 0.033 \\
\hline$\left(Y_{i}-\left(Y_{j}-S_{j}\right)\right) \times$ Treat B $\times$ Treat C & & & & 0.060 & $0.15^{*}$ & -0.021 \\
\hline$\left(Y_{i}-\left(Y_{j}-S_{j}\right)\right) \times$ PseudoTreat B & & & & 0.025 & -0.032 & $0.098 *$ \\
\hline$\left(Y_{i}-\left(Y_{j}-S_{j}\right)\right) \times$ PseudoTreat C & & & & -0.029 & $-0.11 * *$ & 0.035 \\
\hline$\left(Y_{i}-\left(Y_{j}-S_{j}\right)\right) \times$ Round & & & & 0.015 & $0.030^{\star *}$ & -0.0058 \\
\hline$\left(Y_{i}-\left(Y_{j}-S_{j}\right)\right) \times$ Weaker Network & & & & -0.031 & -0.0092 & -0.011 \\
\hline
\end{tabular}




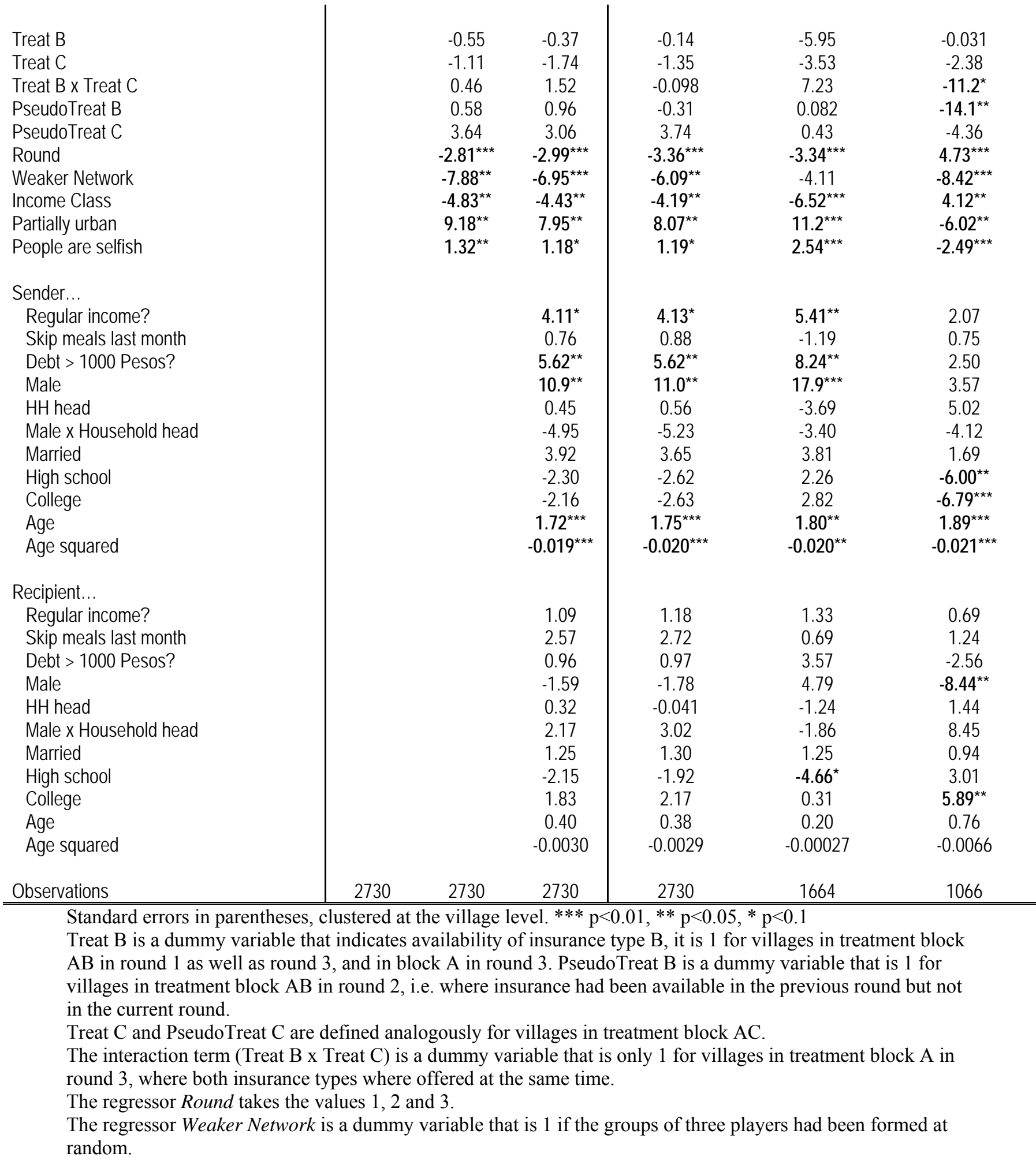

Table A2 complements table 8 and shows the coefficients on the control variables. It seems that men tend to give more. Age has a positive marginal effect until 45 years, when the marginal effect turns negative. Being indebted and regular income are associated with higher latent values. Characteristics of the recipient are mostly insignificant. Regarding the experimental setup participants are less inclined to give in later rounds and in weaker networks. On the community level higher income class (= poorer) is associated with lower 
willingness to transfer, while partially urban communities and (curiously) those that are evaluated as more selfish by the barangay captain exhibit positive coefficients. These effects are all level effects and unrelated to differences in earnings.

Table A3: Descriptive statistics of people who bought insurance in round 1 versus who did not

\begin{tabular}{l|cc|cc}
\hline \hline & Treatment block AB & Treatment block AC \\
& $\begin{array}{c}\text { not insured } \\
(\mathrm{N}=97)\end{array}$ & $\begin{array}{c}\text { insured } \\
(\mathrm{N}=65)\end{array}$ & $\begin{array}{c}\text { not insured } \\
(\mathrm{N}=87)\end{array}$ & $\begin{array}{c}\text { insured } \\
(\mathrm{N}=72)\end{array}$ \\
\hline Male & 0.29 & 0.28 & 0.30 & 0.42 \\
Household head & 0.28 & 0.32 & $\mathbf{0 . 3 1}$ & $\mathbf{0 . 4 4 ^ { * }}$ \\
Married & 0.79 & 0.80 & 0.76 & 0.86 \\
High school education & 0.46 & 0.51 & 0.34 & 0.40 \\
College education & 0.28 & 0.32 & 0.20 & 0.24 \\
Age (in years) & 41.5 & 41.5 & 45.4 & 43.0 \\
Regular monetary income? (dummy) & 0.26 & 0.23 & 0.21 & 0.24 \\
Skip meals in last month & 0.23 & 0.26 & 0.34 & 0.38 \\
In debt with more than 1000 Pesos? & 0.67 & 0.61 & $\mathbf{0 . 4 5}$ & $\mathbf{0 . 6 0}$ \\
\hline
\end{tabular}

Stars indicate significance level of Wilcoxon ranksum test for different means comparing insured to non-insured $* * * \mathrm{p}<0.01, * * \mathrm{p}<0.05, * \mathrm{p}<0.1$

Table A4: Descriptive statistics of people who bought insurance in round 3 versus who did not

\begin{tabular}{l|cc|cc}
\hline \hline & \multicolumn{2}{|c|}{ Treatment block AB } & Treatment block AC \\
& $\begin{array}{c}\text { not insured } \\
(\mathrm{N}=97)\end{array}$ & $\begin{array}{c}\text { insured } \\
(\mathrm{N}=65)\end{array}$ & $\begin{array}{c}\text { not insured } \\
(\mathrm{N}=87)\end{array}$ & $\begin{array}{c}\text { insured } \\
(\mathrm{N}=72)\end{array}$ \\
& Mean & Mean & Mean & Mean \\
\hline Male & 0.29 & 0.28 & 0.31 & 0.40 \\
Household head & 0.30 & 0.30 & 0.35 & 0.40 \\
Married & $\mathbf{0 . 8 5}$ & $\mathbf{0 . 7 0 * *}$ & 0.76 & 0.85 \\
High school education & 0.44 & 0.56 & 0.36 & 0.38 \\
College education & 0.33 & 0.23 & 0.18 & 0.25 \\
Age (in years) & 42.0 & 40.5 & 45.0 & 43.6 \\
Regular monetary income? (dummy) & 0.24 & 0.26 & 0.19 & 0.25 \\
Skip meals in last month & $\mathbf{0 . 1 6}$ & $\mathbf{0 . 3 9 * *}$ & $\mathbf{0 . 2 8}$ & $\mathbf{0 . 4 2}$ \\
In debt with more than 1000 Pesos? & 0.65 & 0.65 & $\mathbf{0 . 4 5}$ & $\mathbf{0 . 5 8 *}$ \\
\hline
\end{tabular}

Stars indicate significance level of Wilcoxon ranksum test for different means comparing insured to non-insured $* * * \mathrm{p}<0.01, * * \mathrm{p}<0.05, * \mathrm{p}<0.1$ 
Table A5 shows alternative specifications of the Tobit regressions and complements Tables 8 and 9. It shows that it does not make a large difference whether we control for village-round fixed effects or for a larger set of controls, and whether we cluster standard errors at the village level, the individual level or whether we obtain the corresponding bootstrap estimate.

Column (1) of table A5 is the same as specification (1) in table 9. Column (5) is the same as specification (2) in table 9. Columns (2) and (6) in Table A5 add village-round fixed-effects. Most of the significant coefficients remain unchanged, particularly those most interesting to our analysis: The interaction terms which reflect crowding out and persistence. It shows that our regressions are not sensitive to the inclusion of further controls on the village-round level.

In columns (3) and (4) we use the same specification as in (1), but different methods for inference. (Hence, the estimated coefficients are all the same.) While in column (1) we used asymptotic standard errors clustered at the village level, column (3) uses clustering at the individual level. In column (4) we estimate standard errors using 1000 bootstrap replications, where the unit of resampling is the individual (i.e. all three rounds together are re-sampled). (Columns (7) and (8) are analogously for the villages without secret saving option.) Overall, the different methods to obtain standard errors lead to rather similar results, with the bootstrap standard errors tending to be a little larger. Thus it is at least as difficult to obtain significant results of the simulation as with error clusters on the village level.

This comparison of the different methods to obtain standard errors is important for the following reason. In Section IV we examined various simulations of poverty lines. The simulation results depend on the estimated $\beta$ coefficients, in a complicated way such that asymptotic standard errors would be difficult to obtain. We therefore use bootstrap methods to obtain some information about the statistical variability of the simulations of Section IV. Since the number of villages is too small for resampling villages, we have to resample individuals, thereby missing any within-village correlations. The results of Table A5 indicate that any within-village correlations do not appear sufficiently large to have a big impact on the variance of the estimated $\beta$ coefficients. (Further details for the simulation procedure are given in appendix II: The simulation is repeated for different parameter estimates to obtain confidence bounds, each of which stems from bootstrap repetitions. To adequately translate clustered standard errors on the village level, one should cluster the bootstrap on the same level. Yet, with such large clusters we have too few clusters for the bootstrap. Instead one can cluster the bootstrap procedure on the individual level (two transfer decisions times three rounds per participant), where we are left with enough clusters.) 
Table A5: Tobit regressions explaining transfers

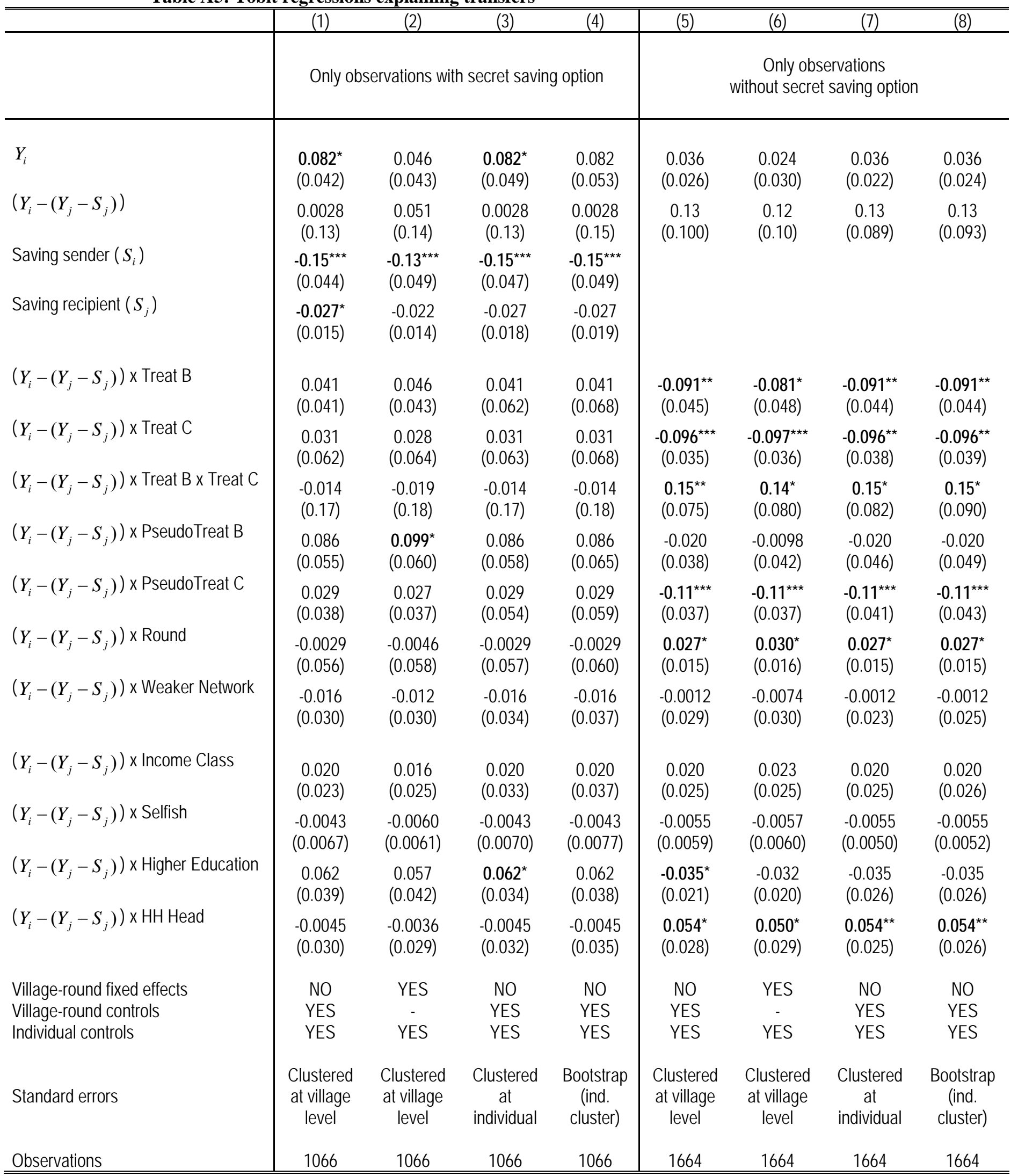

Standard errors in parentheses, clustered at the village or individual level or obtained via clustered (individual level) bootstrap (1000 repetitions), $* * * \mathrm{p}<0.01, * * \mathrm{p}<0.05, * \mathrm{p}<0.1$ 
The following Figure A1 complements Table 10. In Figures A1a and A1b we show the complete distributions of final payoffs under different insurance schemes. The distributions are obtained using the simulation procedure described in appendix II. Figure Ala compares the benchmark of no insurance with availability of insurance without the secret saving mechanism (treatment $\mathrm{AB}$ : availability of type $\mathrm{B}$; treatment $\mathrm{AC}$ : availability of type $\mathrm{C}$ ). The lower tails of the three distributions are very similar and the cumulative distribution functions with insurance are even slightly above the one without insurance in the left tail. Hence, there is no evidence that insurance availability decreases poverty risk.

Figure $\mathrm{A} 1 \mathrm{~b}$ repeats the simulation exercise with the secret saving device in place. The lower tail of the CDF without insurance visibly increases as compared to the figure A1a. This means that the possibility of secret saving increases poverty risk. Different to figure Ala, however, it clearly decreases with both insurance types in the range between 20 and 80 pesos. Table 10 shows specific poverty rates under different schemes for (arbitrary) poverty lines of 40, 50 and $60 \mathrm{PhP}$.

The reason for these findings is that the informal insurance system seems to work rather well in the absence of secret saving. The poverty rates with the formal insurance option $\mathrm{C}$ are the same as without any formal insurance option. An expensive insurance product (i.e. option B) may only do harm, because any additional protection is cancelled out by the crowding out effect on informal solidarity transfers. In contrast, when secret saving is possible, solidarity is low, no crowding out effect of insurance can be observed and insurance is effective in reducing vulnerability.

Figures $\mathrm{A} 2 \mathrm{a}$ and $\mathrm{A} 2 \mathrm{~b}$ complement Table 12 and show the complete distributions of final payoffs under different insurance schemes when assuming no crowding out effects of insurance. Figures $\mathrm{A} 3 \mathrm{a}$ and $\mathrm{A} 3 \mathrm{~b}$ complement Table 13 and show the distributions when assuming mandatory insurance with full take-up. 
Figure A1: CDF of payoff under different insurance schemes a. without secret saving possibility

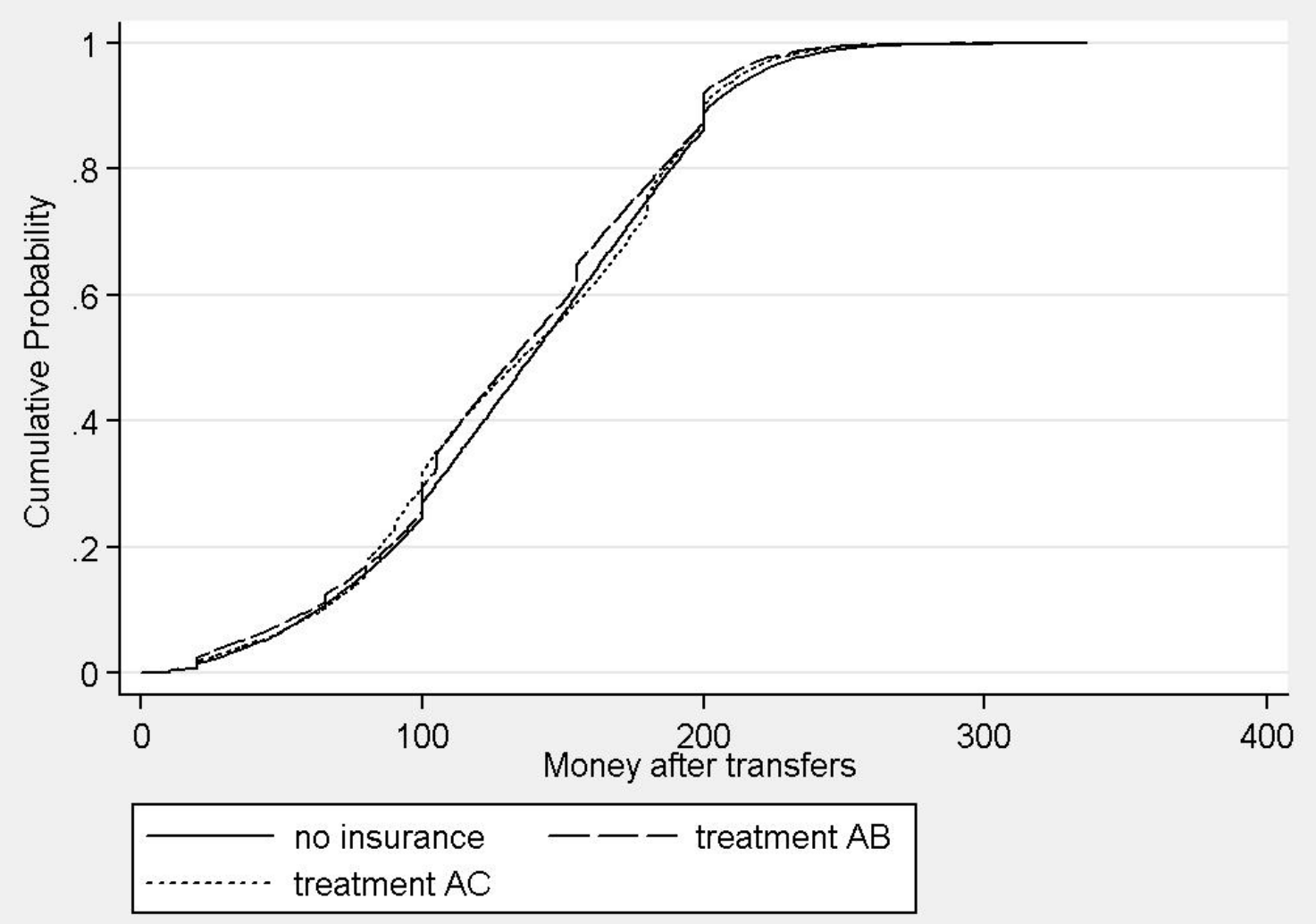

b. with secret saving possibility

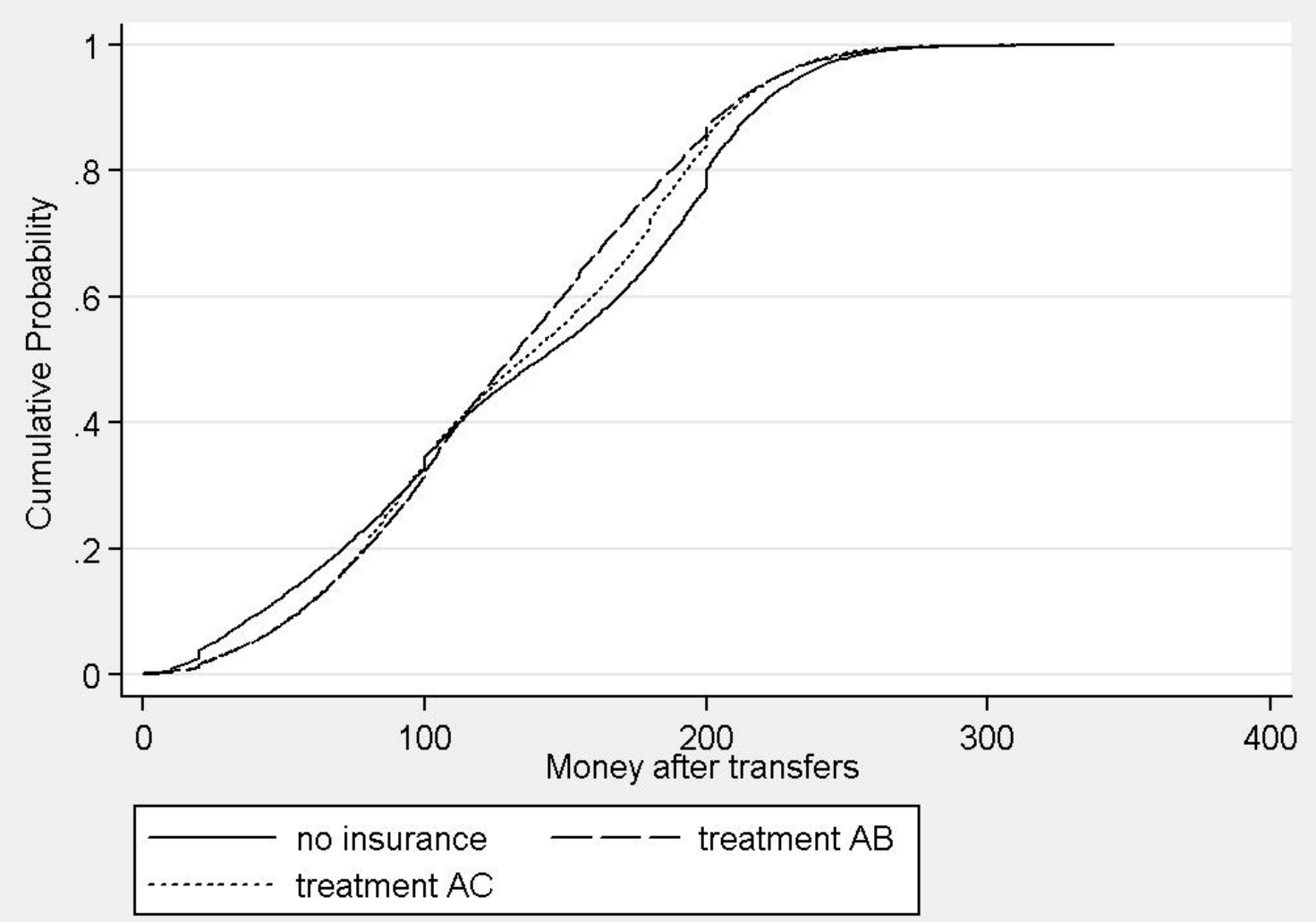


Figure A2: CDF of payoff under different insurance schemes -no crowding out effect of insurance a. without secret saving possibility

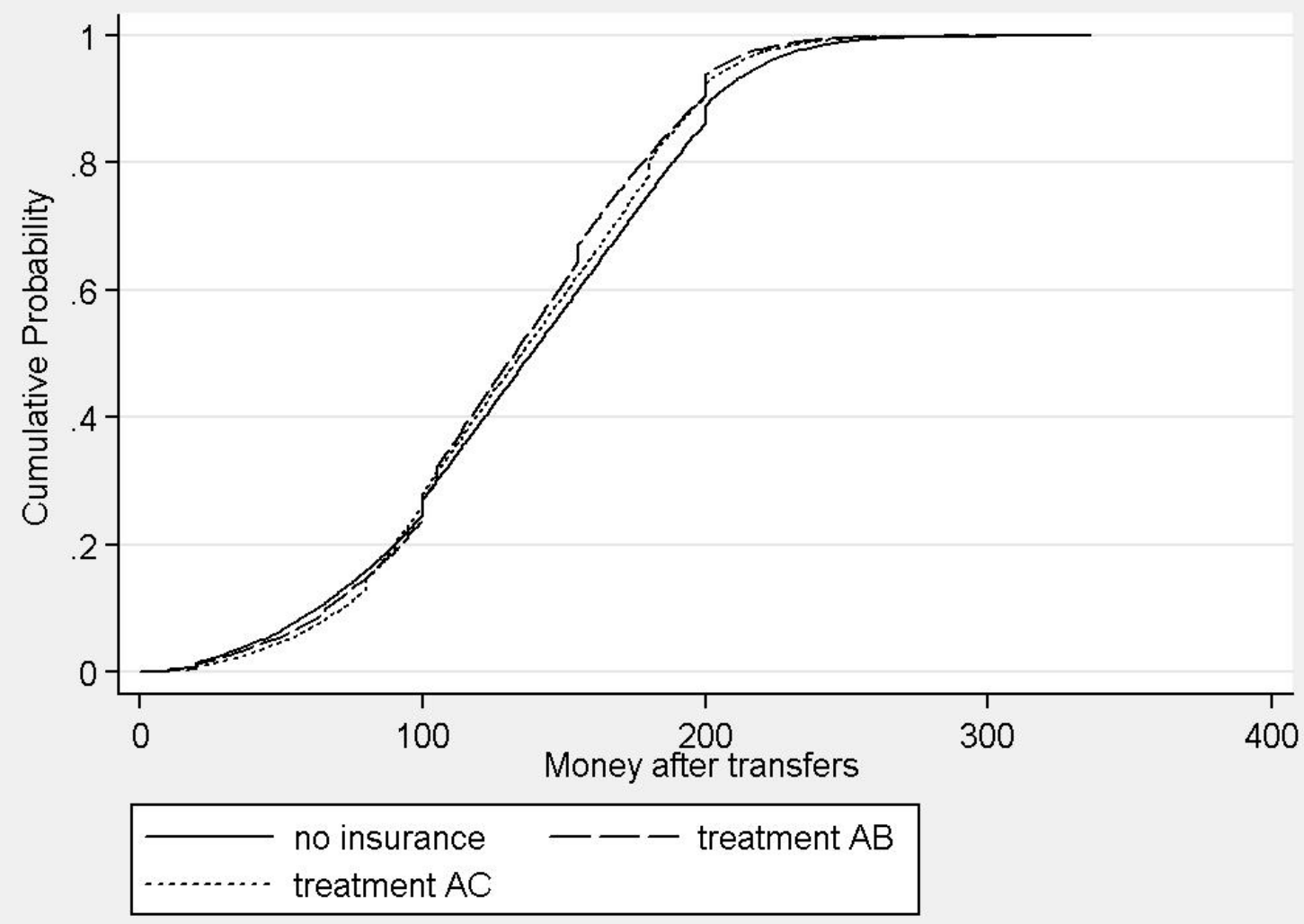

b. with secret saving possibility

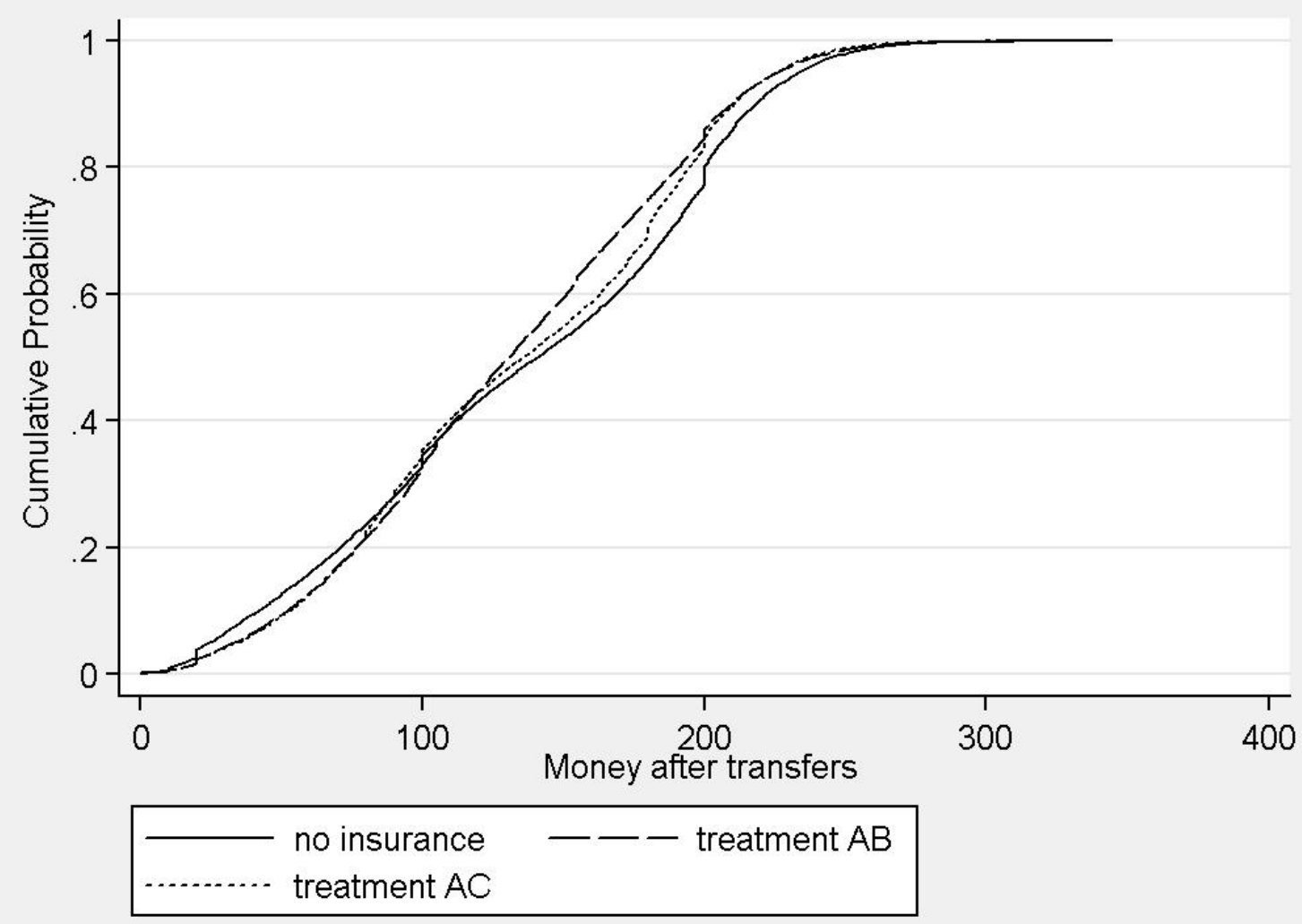


Figure A3: CDF of payoff under different insurance schemes -mandatory insurance a. without secret saving possibility

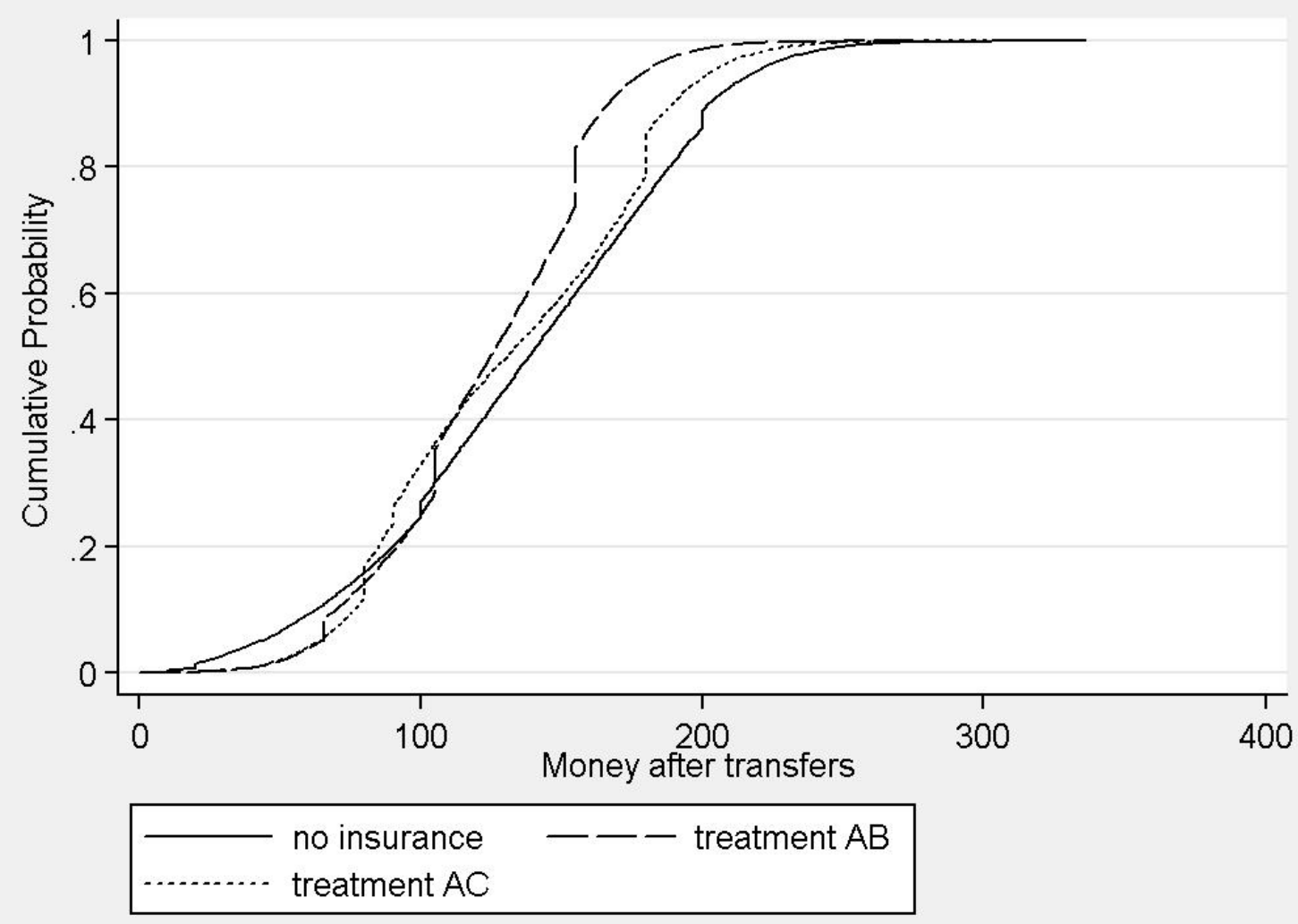

b. with secret saving possibility

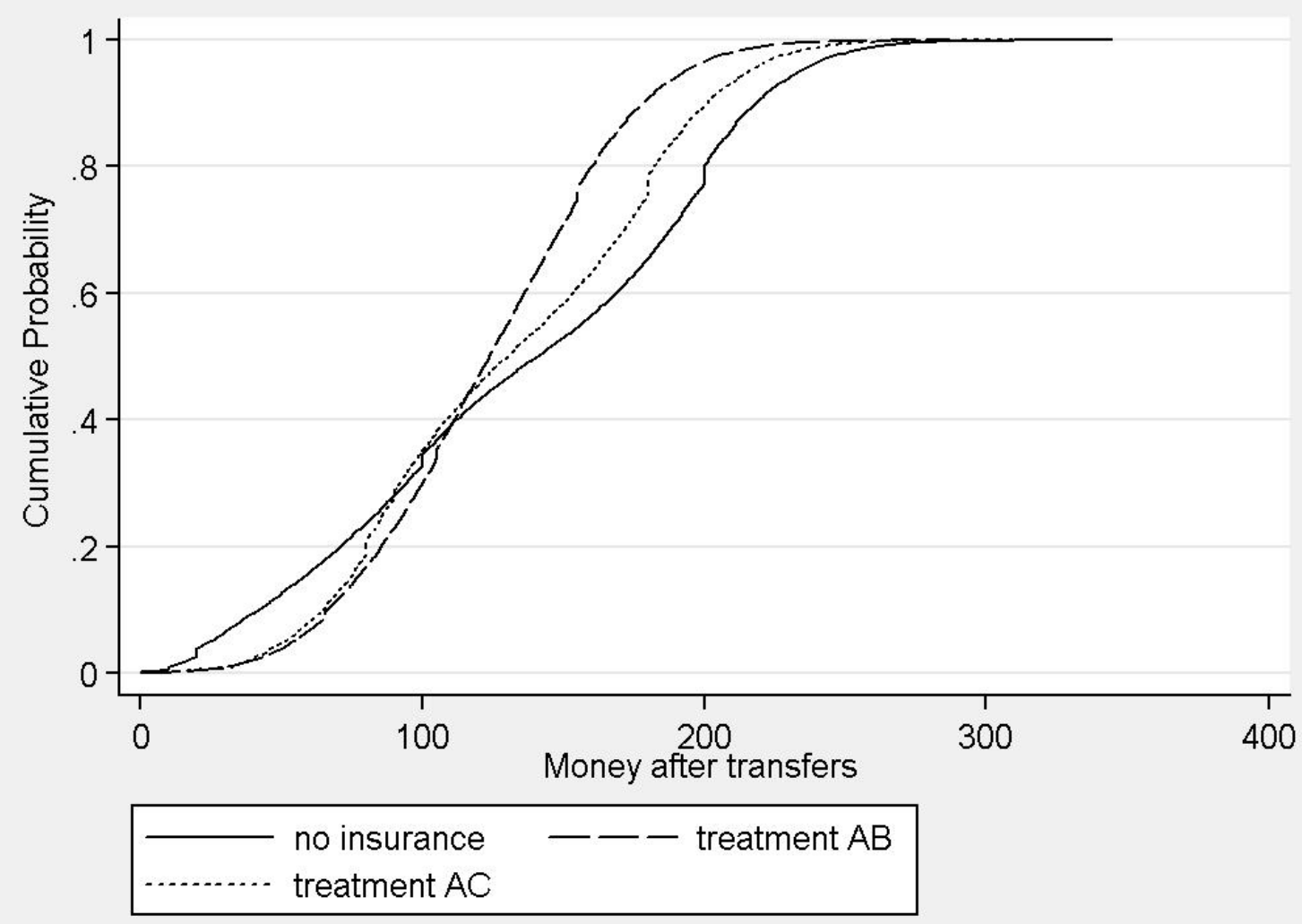




\section{Appendix II: Simulation of transfers under different insurance regimes}

For the simulations we estimate the models with and without the secret saving device separately. Our reference regressions are models (1) and (2) from table 9. The details of the simulation protocol are described below.

In addition to simulating the poverty rates under different scenarios we would also like to know about the statistical variability of these estimated poverty rates. Given the complex simulation procedure, their asymptotic distribution is not trivial. As an alternative, noting that all estimation and simulation steps we use are "smooth", we use the bootstrap to obtain confidence bounds for the simulated poverty rates, by bootstrapping the entire estimation and simulation process. Unfortunately, the number of villages is rather small, such that resampling entire villages could lead to very noisy estimates of the confidence intervals. Instead, we resample individuals. This means we resample at the individual level, i.e. always bundling six transfer observations (two recipients in three rounds for each participant).

Resampling individuals will imply that any within-village correlation structure in the willingness-to-give will get lost. Such possible within-village correlation structures, however, did not appear to have major impacts on the regressions as witnessed in Table A5. There we had analyzed how difference inference methods would change estimated standard errors for the willingness-to-give regressions. We found that asymptotic standard errors with clustering at the village level, asymptotic standard errors with clustering at the individual level as well as bootstrapping at the individual level led to rather similar results, such that we use bootstrap resampling at the individual level for the simulated poverty lines.

The following protocol describes the simulation of the poverty rates. After having estimated the coefficients, we draw random shocks and simulate transfer behavior. In order to eliminate the variance caused by drawing random numbers (shocks, error terms for willingness to transfer), we repeat this process many times. (The actual implementation of repeating this many times was done by expanding the original dataset 100 times.) This delivers an estimate of the expected poverty rate. For estimating the variability of this estimate, the entire simulation protocol is bootstrapped. 


\section{$\underline{\text { Simulation protocol: }}$}

1. Estimate model of transfers:

We estimate regression model (1) and (2) from table 9 for the sample with/without secret savings. All coefficients are stored.

2. Expand data:

For the results to be stable and to represent average treatment effects, we have to create a sufficiently large data set. We therefore extend the whole data set 100 times (including observations both with and without secret saving possibility).

3. Draw shock:

For each (participant-round) observation we draw a shock according to the theoretical probabilities ( $1 / 2$ no shock, 1/3 medium shock, 1/6 catastrophic shock).

4. Draw 'risk aversion':

To know which individual will choose insurance in our simulation, we draw an individual-round specific risk aversion parameter from a uniform distribution between 0 and 1. If risk aversion is above a certain threshold, the individual will be treated as having chosen insurance in the insurance treatments. The thresholds are chosen such that the take-up rates of type $\mathrm{B}$ and type $\mathrm{C}$ equal the observed take-up rates in the experiment. (This is obviously a simplification, but in the game we observe that takeup in the first round is no perfect predictor of take-up in round three. $)^{53}$

5. Draw hiding propensity (if applicable):

$94 \%$ of participants secretly save if they have the possibility to do so. This is also the probability in the simulation procedure.

6. Draw error term

We draw an error term from the normal distribution with the estimated variance from the regression model (1) and (2) of table 9. This is an independent term for each (individual-round-recipient) observation.

7. Simulate setting for each regime (A vs. AB vs. $A C)$

a. Reset insurance treatment indicators according to setting

b. Assign insurance to the risk-averse individuals (see step 4)

c. Assign loss/payoff according to shock (see above) and insurance 'choice' (this is the pre-transfer outcome)

\footnotetext{
${ }^{53}$ We could explicitly estimate a model to explain insurance take-up, conditional on the covariates. However, this would very much complicate our exercise. Also, distribution is only affected by this simplification if there are substantial factors connected to insurance take-up and redistributive preferences at the same time.
} 
d. Assign secret saving (if applicable)

e. Using the previously generated random variables, calculate all the regressors displayed in Table 9

f. Predict transfers, using the $\beta$ coefficients estimated in step 1 and adding the error term of step 6

g. Left-censor transfers (according to Tobit specification) and right-censor at half of post-lottery income. (We censor at half of the income because each player can make transfers to two co-players. By censoring at half of the income we ensure that the total transfers made to both players are at most $100 \%$ of the income. I.e. in our design, people cannot transfer more money than what they have.)

h. From the post-lottery income and censored transfers calculate post-transfer incomes.

The income/loss distribution from each setting can now be further analyzed (e.g. for poverty rates, etc.). To obtain confidence bounds for the distribution, coefficients of the reference model are estimated repeatedly with new bootstrap samples (clustered at the individual level). With each bootstrap estimation, the simulation is repeated. 


\section{Appendix III: Differences in crowding-out between insurance types}

In table 9 we studied subsets of those participants where secret saving was not possible as a robustness check. We examined the effects separately for a 'high understanding' subsample (column 3), for rounds one and two (column 4), and rounds two and three (column 5). The interaction coefficients are significant in two out of three cases for the more general insurance type B, but this is not the case for catastrophic-insurance type C. While effects always point in the expected (negative) direction, they are insignificant and smaller than for insurance type B. This raises the question whether the crowding-out effect of insurance type $\mathrm{C}$ is systematically smaller, or whether this is simply random variation. We therefore use round 1 and round 3 of treatment blocks $\mathrm{AB}$ and $\mathrm{AC}$ to directly compare the sensitivity to differences between both treatment blocks. ${ }^{54}$ Note that we are in the case without secret saving, so $S_{i}, S_{j}$ do not play a role, because they are both zero by design. Therefore the following table contains the regressor $\left(Y_{i}-Y_{j}\right)$ instead of $\left(Y_{i}-\left(Y_{j}-S_{j}\right)\right)$.

Table A6 shows compares the sensitivity to differences between treatment B and C in round 1 (column 1), round 3 (column 2) and pooled in round 1+3 (column 3). The interaction $\left(Y_{i}-Y_{j}\right) \times$ Treat $B$ indicates differences in the crowding-out effect. Du to the limited sample size they are all insignificant. In specification (1) there is a substantial negative interaction effect, indeed indicating that in the first round the crowding-out of solidarity is larger for treatment B. In specification (2), however, the sign of the coefficient is reversed. Pooling the two rounds results in a very low size of the interaction coefficient. This suggests that there is no difference between the two crowding-out effects and that variation in the interactions across rounds is purely random.

Table A6: Tobit regressions explaining transfers - treatment B vs. C

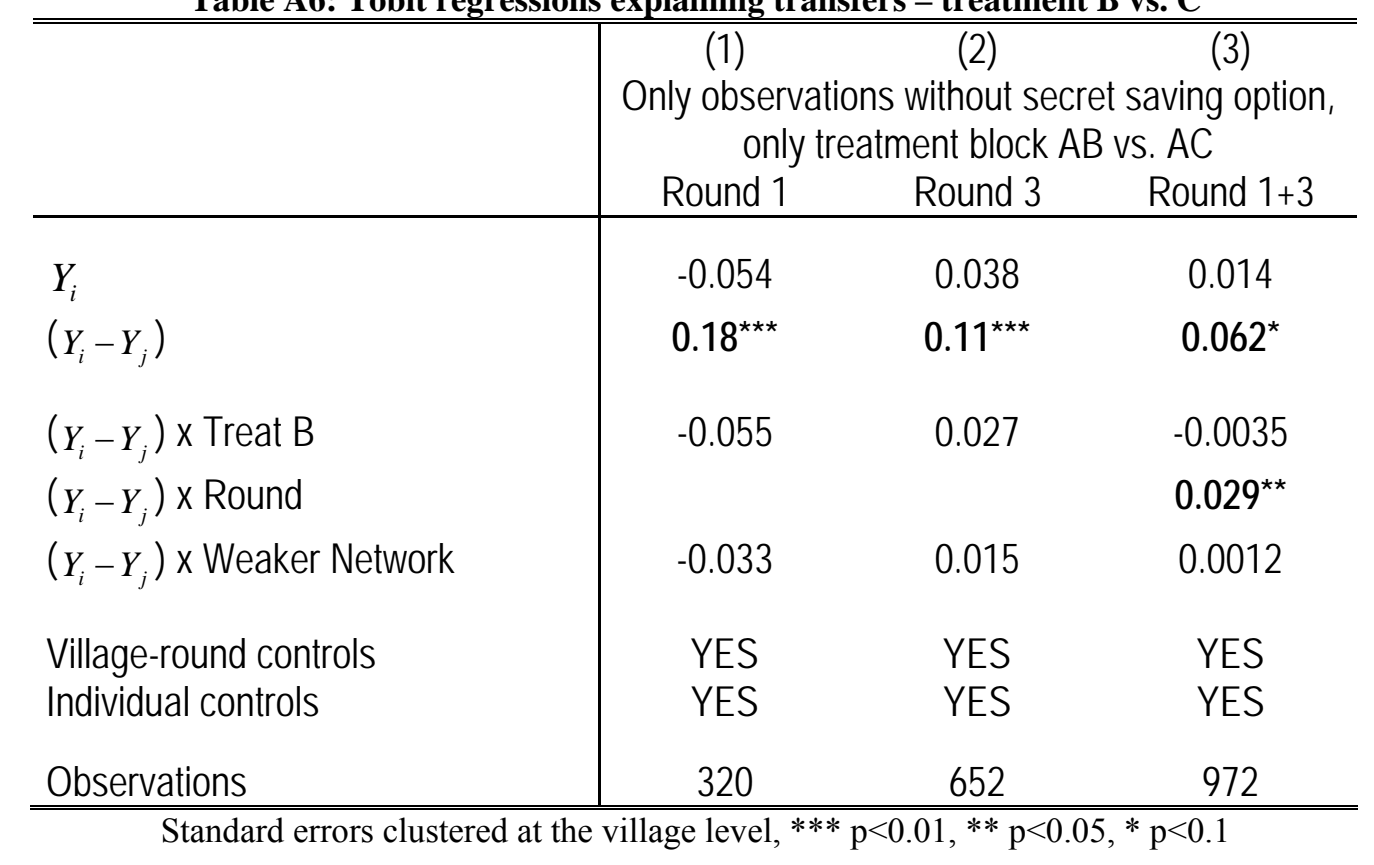

\footnotetext{
${ }^{54}$ Evaluate table 2 (treatment plan) to see that the experimental setup is perfectly balanced in this comparison.
} 


\section{Appendix IV: Test Questionnaire}

(Notes: Example for treatment block $\mathrm{AB}$, round 1, with saving. In reality we called option $\mathrm{A}$

"Angola", B "Botswana" and C "Cameroon" to avoid a notion of order in the options. Correct answers given.)

When do you decide which option you choose?

$\bigotimes 1$ before you throw the dice

2 after you throw the dice

$\square 3$ whenever you like

CORRECT? YES $\square$ NO

Is the option BOTSWANA for free?

YES $\square \quad$ NO $\mathbb{Q}$

CORRECT? YES $\square$ NO

How much does the option BOTSWANA cost?

45

CORRECT? YES $\square$ NO

How much do you have left if...

\begin{tabular}{|l|c|c|}
\hline & With option BOTSWANA & With option ANGOLA \\
\hline$\ldots$ you roll a 1? & $\mathbf{1 5 5}$ & $\mathbf{2 0 0}$ \\
\hline$\ldots$ you roll a 2? & 155 & 200 \\
\hline$\ldots$ you roll a 3? & 155 & $\mathbf{2 0 0}$ \\
\hline$\ldots$ you roll a 4? & 105 & $\mathbf{1 0 0}$ \\
\hline$\ldots$ you roll a 5? & $\mathbf{1 0 5}$ & $\mathbf{1 0 0}$ \\
\hline$\ldots$ you roll a 6? & $\mathbf{6 5}$ & $\mathbf{2 0}$ \\
\hline
\end{tabular}

ONLY IF WITH LOCKBOX

When can you put money in the lockbox? Can you put money in the lockbox if you choose option ANGOLA and...

... you roll a 1 ?

... you roll a 2 ?

... you roll a 3?

... you roll a 4?

... you roll a 5?

... you roll a 6 ?

\begin{tabular}{|c|c|c|}
\hline YES $\boldsymbol{Z}$ & & NO [ \\
\hline YES & & \\
\hline & & \\
\hline ES & & \\
\hline $\mathrm{S}$ & & \\
\hline IES & & \\
\hline
\end{tabular}

If yes, how much

100

If yes, how much

If yes, how much

If yes, how much

If yes, how much

If yes, how much

\begin{tabular}{l}
100 \\
\hline 100 \\
\hline 100 \\
\hline \\
\hline
\end{tabular}

CORRECT? YES $\square$ NO

When can you put money in the lockbox? Can you put money in the lockbox if you choose option BOTSWANA and...

... you roll a 1 ?

... you roll a 2 ?

... you roll a 3 ?

... you roll a 4 ?

... you roll a 5 ?

... you roll a 6 ?

\begin{tabular}{|c|c|}
\hline YESZ & NO \\
\hline YES & \\
\hline YES & \\
\hline YES & \\
\hline YES & \\
\hline & \\
\hline
\end{tabular}

If yes, how much

If yes, how much

If yes, how much

If yes, how much

If yes, how much

If yes, how much

\begin{tabular}{l}
50 \\
\hline 50 \\
\hline 50 \\
\hline \\
\hline
\end{tabular}

CORRECT? YES $\square$ NO

Will your group members know if you put money in the lockbox?

YES $\square \quad$ NO $\square$ 